\title{
Maltodextrin-Based Carbohydrate Oral Rinsing and Exercise Performance: Systematic Review and Meta-Analysis
}

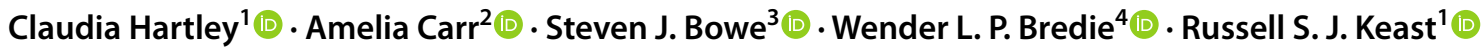

Accepted: 1 February 2022 / Published online: 3 March 2022

(c) The Author(s) 2022

\begin{abstract}
Background Carbohydrates are an important fuel for optimal exercise performance during moderate- and high-intensity exercise; however, carbohydrate ingestion during high-intensity exercise may cause gastrointestinal upset. A carbohydrate oral rinse is an alternative method to improve exercise performance in moderate- to high-intensity exercise with a duration of 30-75 min. This is the first systematic review and meta-analysis to comprehensively examine the isolated effect of maltodextrin-based rinsing on exercise performance.

Objective The objective of this review was to establish the effect of a maltodextrin-based carbohydrate oral rinse on exercise performance across various modes of exercise. Furthermore, a secondary objective was to determine the effects of moderators [(1) participant characteristics; (2) oral rinse protocols; (3) exercise protocol (i.e. cycling, running etc.) and (4) fasting] on exercise performance while using a maltodextrin-based, carbohydrate oral rinse.

Methods Five databases (MEDLINE, PsycINFO, Embase, SPORTDiscus and Global Health) were systematically searched for articles up to March 2021 and screened using Covidence (a systematic review management tool). A random effects robust meta-analysis and subgroup analyses were performed using Stata Statistical Software: Release 16.

Results Thirty-five articles met the inclusion criteria and were included in the systematic review; 34 of these articles were included in the meta-analysis. When using a conventional meta-analytic approach, overall, a carbohydrate oral rinse improved exercise performance in comparison with a placebo ( $\mathrm{SMD}=0.15,95 \% \mathrm{CI} 0.04,0.27 ; p=0.01$ ). Furthermore, when implementing an adjusted, conservative, random effects meta-regression model using robust variance estimation, overall, compared with placebo, a carbohydrate oral rinse demonstrated evidence of improving exercise performance with a small effect size $(\mathrm{SMD}=0.17,95 \% \mathrm{CI}-0.01,0.34 ; p=0.051)$.

Conclusion This systematic review and meta-analysis demonstrates that a maltodextrin-based carbohydrate oral rinse can improve exercise performance. When comparing the two meta-analytic approaches, although non-significant, the more robust, adjusted, random effects meta-regression model demonstrated some evidence of a maltodextrin-based carbohydrate oral rinse improving exercise performance overall.
\end{abstract}

\section{Introduction}

In the 1920s, it was recognised that carbohydrates were a crucial fuel source for exercise [1] with improved exercise capacity and general exercise performance being linked to carbohydrate consumption [2]. In subsequent research, it was demonstrated that a glucose polymer solution improved exercise capacity during cycling time to exhaustion tests compared with a placebo solution [3, 4]. Similar findings indicated that time to fatigue was significantly longer after

Russell S. J. Keast

russell.keast@deakin.edu.au

Extended author information available on the last page of the article ingesting a glucose polymer solution and glucose infusion in comparison with a placebo [3]. Currently, it is standard practice for individuals to ingest carbohydrates prior to or during sustained high intensity or endurance exercise [5-8]. Based on literature by Burke et al. [9], the American College of Sports Medicine (ACSM) recommends the total daily intake of 5-7 g per kilogram per day of carbohydrates for a moderate daily exercise programme (i.e. $1 \mathrm{~h} /$ day); which can be ingested either prior to exercise, during exercise or in recovery from a previous exercise session [9-11]. The ACSM details further recommendations for acute fuelling strategies for carbohydrate loading, pre-event fuelling and for during brief exercise ( $<45 \mathrm{~min}$ ), sustained high-intensity exercise (i.e. $45-75 \mathrm{~min}$ ) and endurance exercise (1-2.5 h). 


\section{Key Points}

According to the robust, meta-regression model, a maltodextrin-based carbohydrate oral rinse shows some evidence of improving exercise performance.

According to a conventional meta-analytic approach, rinsing a maltodextrin-based carbohydrate oral rinse for $10 \mathrm{~s}$ with a concentration between 6 and $6.5 \%$ is effective at improving exercise performance.

During sustained high-intensity exercise, it is currently recommended that small amounts of carbohydrates be ingested (including mouth rinsing) for optimal carbohydrate intake, whereas during endurance exercise, it is recommended that $30-60 \mathrm{~g}$ of carbohydrates be ingested per hour $[9,10]$. A potential disadvantage associated with carbohydrate ingestion, however, is the possible occurrence of gastrointestinal discomfort $[6,12-15]$, which can subsequently negatively affect exercise performance [16-18].

An alternative method to utilise carbohydrates during exercise is a carbohydrate oral rinse. Previous research indicates that a maltodextrin rinse comprising of a $6-6.4 \%$ maltodextrin-based solution [19-21] during moderate- to high-intensity exercise with a time span ranging from 30 to 75 min can facilitate improvements in exercise $[6,8]$. The exact mechanism that facilitates improvements in exercise performance after a carbohydrate oral rinse remains unknown. It is proposed that alterations in exercise performance may be influenced by a 'Central Governor' mechanism to maintain homeostasis during exercise [22]. The 'Central Governor' is thought to modify power output through the use of afferent signals from peripheral physiological receptors and systems that detect changes in the external and internal environment [22]. Therefore, it could be interpreted that during exercise, the positive central responses to a carbohydrate oral rinse could possibly counteract the negative physical, metabolic and thermal afferent signals [23]. An alternative theory is that improved exercise performance is a result of enhanced brain activation in higher brain regions. It is thought that these higher brain regions link the corresponding cognitive, behavioural and emotional response and the gustatory pathways [24, 25]. Furthermore, these regions have been found to be activated by oral exposure to carbohydrates but not by non-nutritive sweeteners [19, 26, 27], which may assist in explaining the positive effects of carbohydrate rinsing on exercise performance.

Carter et al. [28] first investigated the effects of a carbohydrate oral rinse on performance during a cycling time trial. During the time trial, participants were instructed to complete a certain amount of work ( $\mathrm{kJ}$ ) as quickly as possible. This amount of work was based on a formula including each participant's maximum power output value $\left(W_{\max }\right)$ [28]. During the time trial either a $6.4 \%(\mathrm{w} / \mathrm{v})$ maltodextrin or water (placebo) sample was rinsed in the mouth for 5 $\mathrm{s}$ prior to expectoration. With the carbohydrate oral rinse, performance time was significantly faster $(2.9 \%)$ in comparison with the water rinse (placebo) [28]. Additionally, improvements in exercise performance after an oral carbohydrate rinse in comparison with a placebo rinse have been found with cycling [29-33], running [34-37] and resistance exercise [38]. In contrast, some studies have reported no significant improvements in exercise performance [39-46]. This lack of significant improvements may be due to the study design (i.e. mode of exercise, concentration and/or composition of the rinse and rinsing duration) or lack of statistical power to detect changes. Due to the inconsistent results in the pool of literature, further analysis is required to investigate if a maltodextrin-based oral rinse does improve exercise performance.

Furthermore, research in this area has also discussed a possible placebo effect in conjunction with carbohydrate oral rinsing. As previous research has demonstrated that placebo effects may have a significant impact on physical performance [47], it is common practice for at least two oral rinses to be trialled: a carbohydrate oral rinse and a placebo oral rinse [28, 29]. To minimise possible placebo effects between the rinsing conditions, previous research has also blinded participants to the composition of the rinses and also to the true objective of the experiment [45].

Previous reviews have focused on the effects of carbohydrate oral rinsing on exercise performance across running and cycling performance [48], cycling performance [49] and sprinting performance [50], and carbohydrate oral rinsing alongside ingestion and loading on exercise performance [51]. However, no reviews have specifically discussed the intricacies of the maltodextrin used in the carbohydrate oral rinse. Maltodextrin is a variable starch-based structure [52] that is a widely used product in foods and food manufacturing [53]. Maltodextrin can vary depending on its physical and chemical properties, which can in turn affect the overall flavour and appearance [54]. Additionally, as maltodextrin can vary widely in terms of structure and origin, these may be important factors to investigate as this variation may impact exercise performance. For example, starches are composed of two types of glucose polymers: amylose and amylopectin [55]. The ratio between amylose and amylopectin can affect the physical properties of starches including their retrogradation tendencies, viscosity and pasting properties [56-58]. Other important structural factors include dextrose equivalent (DE) and degree of polymerisation (DP). For example, a shorter-chain maltodextrin has a higher DE 
and lower DP and therefore has a sweeter taste in comparison with a longer-chain maltodextrin [54]. The origin of the maltodextrin may also be an important factor to consider as maltodextrin can be made from corn, rice, manioc, oat or potato starch [59]. Depending on the source of the starch, the ratio of amylose to amylopectin changes. For example, high-amylose corn starch has an amylose content of 50-70\%, whereas potato, tapioca and wheat starches have an amylose content close to $20 \%$ [58]. Without reporting on the type of maltodextrin used in the carbohydrate oral rinse, the information concerning origin and structure is unknown, potentially prohibiting informed observations and mechanistic insights. Furthermore, oral rinse protocol (concentration and duration) is an important factor to investigate as dose response or time/duration response with a carbohydrate oral rinse and exercise performance response may exist. Exercise protocol, fasting and participant characteristics are additional factors that can also vary across the literature and are important to investigate as there may be an optimum level or conditions at which exercise performance can be improved. The primary aim of this systematic review and meta-analysis is to comprehensively examine the isolated effect of maltodextrin-based rinsing on exercise performance. Furthermore, the secondary aim of this review is to investigate the effect of the concentration and composition of the rinse, duration of the rinse and the impact of participant characteristics (i.e. sex), fasting and exercise protocol on exercise performance.

\section{Methods}

The systematic review and meta-analysis was completed according the Cochrane Handbook for Systematic Reviews of Interventions [60] and following the PRISMA (Preferred Reporting Items for Systematic Reviews and Meta-Analyses) statement [61].

\subsection{Eligibility Criteria}

For this systematic review and meta-analysis, studies were included if they met the following inclusion criteria: (1) investigated the effect of a maltodextrin oral rinse on exercise performance; (2) randomised, blinded counterbalanced or crossover, control or placebo study design; (3) a maltodextrin-based carbohydrate oral rinse with a concentration of no less than $6 \%$ being rinsed for a minimum of $5 \mathrm{~s}$ (based on existing literature in the area $[28,49]) ;(4)$ original research articles and (5) human participants. Studies were excluded if they (1) did not use maltodextrin in the oral rinse; (2) involved any ingestion of the maltodextrin rinse; (3) were not original research articles (i.e. conference abstracts, review articles); (4) were not written in English and (5) did not have sufficient methodological information to allow a check of the inclusion criteria. The search included articles that were published up to and including February 2021.

\subsection{Data Sources and Search}

Initially, a small test search using the chosen search terms was conducted to determine the efficacy of the search terms. After the test search confirmed the search strategy was effective, the search terms were finalised. Five separate databases (MEDLINE, PsycINFO, Embase, SPORTDiscus and Global Health) were searched and this was performed initially in June 2020 and updated in March 2021. The following terms were used in the search: Carbohydrate OR CHO OR Carbohydrates $A N D$ Oral Rinse $O R$ Oral Rinsing $O R$ Mouth Rinsing $O R$ Mouth Rinse $O R$ Rinsing $O R$ Rinse $O R$ Mouth Wash $A N D$ Exercise Performance $O R$ Performance $O R$ Exercise $O R$ Cycling $O R$ Running $O R$ Sprinting $O R$ Resistance.

This search strategy yielded 527 publications. The hand searching technique, which involved searching reference lists of included studies and review articles for relevant studies, found a further ten studies. From the pool of included studies, 288 duplicates were removed which resulted in 239 studies for screening. After screening, a total of 35 studies were included for data extraction and analysis for the systematic review and of these 35 studies, a total of 34 studies were included in the meta-analysis. No relevant studies were found within grey literature. The process of study selection and screening is summarised in a PRISMA flow diagram (Fig. 1).

\subsection{Study Selection and Data Collection}

The studies were imported into a systematic review management tool (Covidence, Veritas Health Innovation, Victoria, Australia) to complete the screening process. The titles, abstracts and full texts of the studies were reviewed separately to check for eligibility criteria. The screening process for abstract screening and full-text screening was completed by three separate reviewers. Screening conflicts were resolved through discussion between the three independent reviewers until a conclusion was reached. Data extraction for the included studies focused on (1) title, year of publication, type of publication and (2) methods and design of the study, participants selected (sample size, sex and age of participants), intervention (composition and concentration of oral rinse), type of exercise performed and outcome measures (i.e. distance covered in the trial [m], time to complete the trial $[\mathrm{s}]$, power output $[\mathrm{W}]$, speed $[\mathrm{km} / \mathrm{h}])$. As the majority of studies reported results as mean \pm standard deviation, studies that reported mean \pm standard error were converted to mean \pm standard deviation for consistency. If additional information was required from a study for data extraction, the corresponding author was contacted. On the occasion 


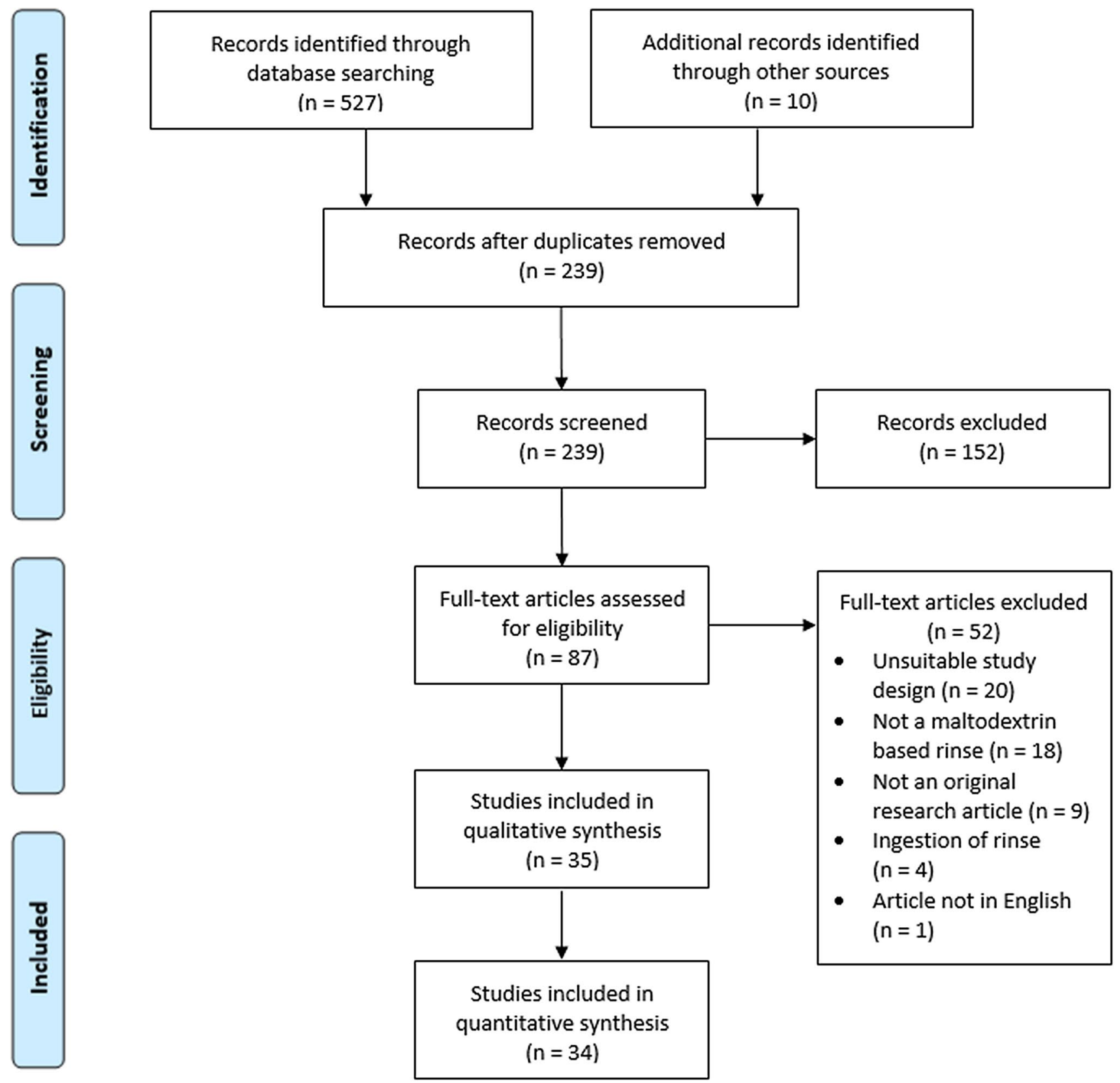

Fig. 1 PRISMA flow diagram [62]

where this further information was unable to be obtained, that study was excluded from the meta-analysis.

\subsection{Quality Assessment}

This quality assessment was completed using a modified version of the Quality Criteria Checklist: Primary Research [63] and previously published formatting [64]. One author (C.H.) performed the quality assessment and a second author (R.S.J.K.) independently cross-checked the quality assessment. Any disagreements were discussed and resolved between the two authors.

\subsection{Risk of Bias Assessment}

A risk of bias assessment was conducted with the studies that were included in the meta-analysis according to the Cochrane Collaboration's recommendation for systematic reviews [65]. The categories for assessment included (1) random sequence generation (selection bias); (2) blinding of participants and personnel (performance bias); (3) blinding 
of outcome assessment (detection bias); (4) incomplete outcome data (attrition bias) and (5) selective reporting (reporting bias). Each category was assessed and assigned either a low risk of bias, high risk of bias or unclear risk of bias. One author (C.H.) performed the risk of bias assessment and a second author (R.S.J.K.) independently cross-checked the risk of bias assessment. Any disagreements were discussed and resolved between the two authors.

\subsection{Statistical Analysis}

The data extraction process for the meta-analysis focused on primary performance-based outcomes (refer to Table 1 for a complete list). Outcomes that were not deemed to be performance based were excluded from the meta-analysis data set. Studies with multiple results for a single performance outcome (i.e. maximal speed: sprint 1 , sprint 2 , sprint 3 etc.) were collapsed and averaged together prior to the meta-analysis. From the 34 included articles, the data collection process resulted in 58 data points for analysis.

\subsubsection{Conventional Meta-Analytic Method-Standardised Mean Differences (SMD)}

The effects of oral rinsing were analysed in terms of means and standard deviations comparing $\mathrm{CHO}$ and placebo (PLA) treatments at the end of the study time. Thus, the standardised mean difference (SMD) was calculated using the Hedges' $g$ method for each individual effect (CHO vs PLA) reported in each study. The Hedges' $g$ method was adjusted using exact computation for the bias-correction factor and Hedges and Oikin were used for standard error for each individual effect size. Conventional meta-analytic techniques rely on the assumption that effect size estimates from different studies are independent and have sampling distributions with known conditional variances [66, 67]. Initially, a conventional random effects (restricted maximum likelihood) meta-analysis model was used to compare the carbohydrate-based oral rinse and placebo rinse conditions. However, this conventional approach was unable to account for the multiple dependent effect sizes (SMDs) from each article included within the current review.

\subsubsection{Robust Variance Estimation (RVE) Meta-Regression}

Subsequently, a random effects meta-regression model using robust variance estimation [67] was used to compare the overall effect of a carbohydrate-based oral rinse on exercise performance. This model technique provides a robust method for estimating standard errors in a meta-regression, particularly when there are dependent effects as was the case in this review. This meta-regression technique was used as a constant-only model and compared with the conventional pairwise meta-analytic method used earlier.

\subsubsection{Moderators}

To explore the effects of moderators, a subgroup analysis was performed on the following moderators: (1) participant characteristics; (2) oral rinse protocols; (3) exercise protocol (i.e. cycling, running etc.) and (4) fasting. Heterogeneity was assessed initially using the $I^{2}$ statistic and a value over $50 \%$ was deemed to represent substantial heterogeneity [60].

\subsubsection{Conventional Subgroup Meta-Analysis and a More Robust Variance Approach}

Similar issues of dependence within subgroups arose, so initially, conventional techniques to explore the potential statistical heterogeneity of the subgroups were used. Random effects meta-regression models using robust variance estimation were then applied to explore the moderators separately. A sensitivity analysis for categorical moderator variables was conducted for groups with less than four studies, by removing the group from the analysis. With fewer than four studies, the small sample adjustments are deemed inaccurate and hence could not be done with this modelling approach [67].

All analyses were performed through the statistical analysis software Stata (StataCorp 2019. Stata Statistical Software: Release 16. College Station, TX, USA: StataCorp LLC), with a significance level set at $5 \%(p<0.05)$. Stata meta-analysis packages used were: meta (for conventional pairwise and subgroup meta-analysis) and robumeta (random effects meta-regression model using robust variance estimation) [67] (https://onlinelibrary.wiley.com/doi/abs/10 . 1002/jrsm.1091).

\section{Results}

\subsection{Systematic Review Results}

\subsubsection{Search Results}

In total, 35 studies were eligible for inclusion in the systematic review and 34 studies were eligible for inclusion in the meta-analysis. The main results and characteristics from the included studies are displayed in Table 1.

\subsubsection{Characterisation of Participants}

Across the studies, the sample size ranged from seven to 21 participants, totalling 444 participants. Of these 444 participants, 380 were males and 64 were females. Of the total 


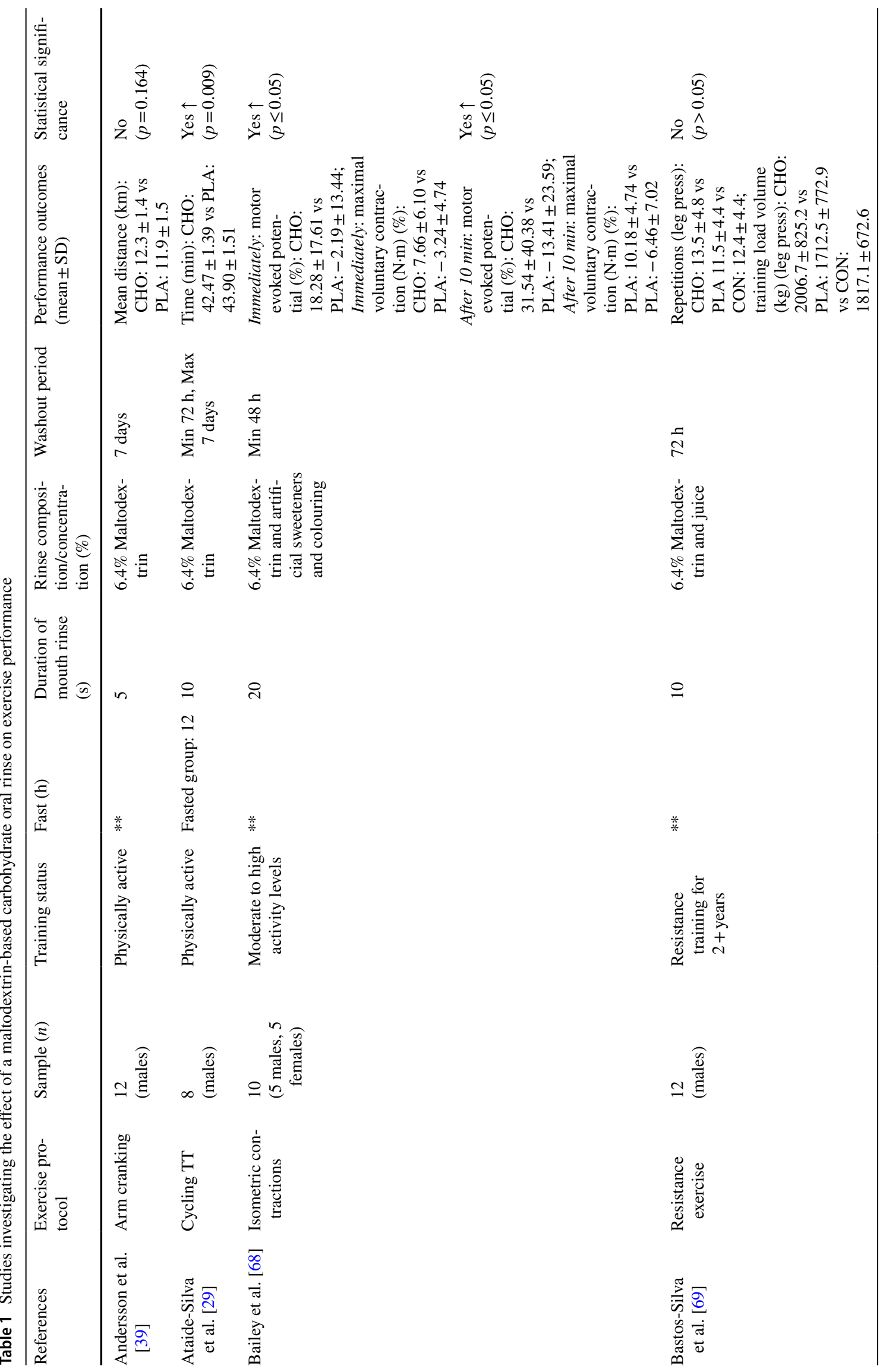




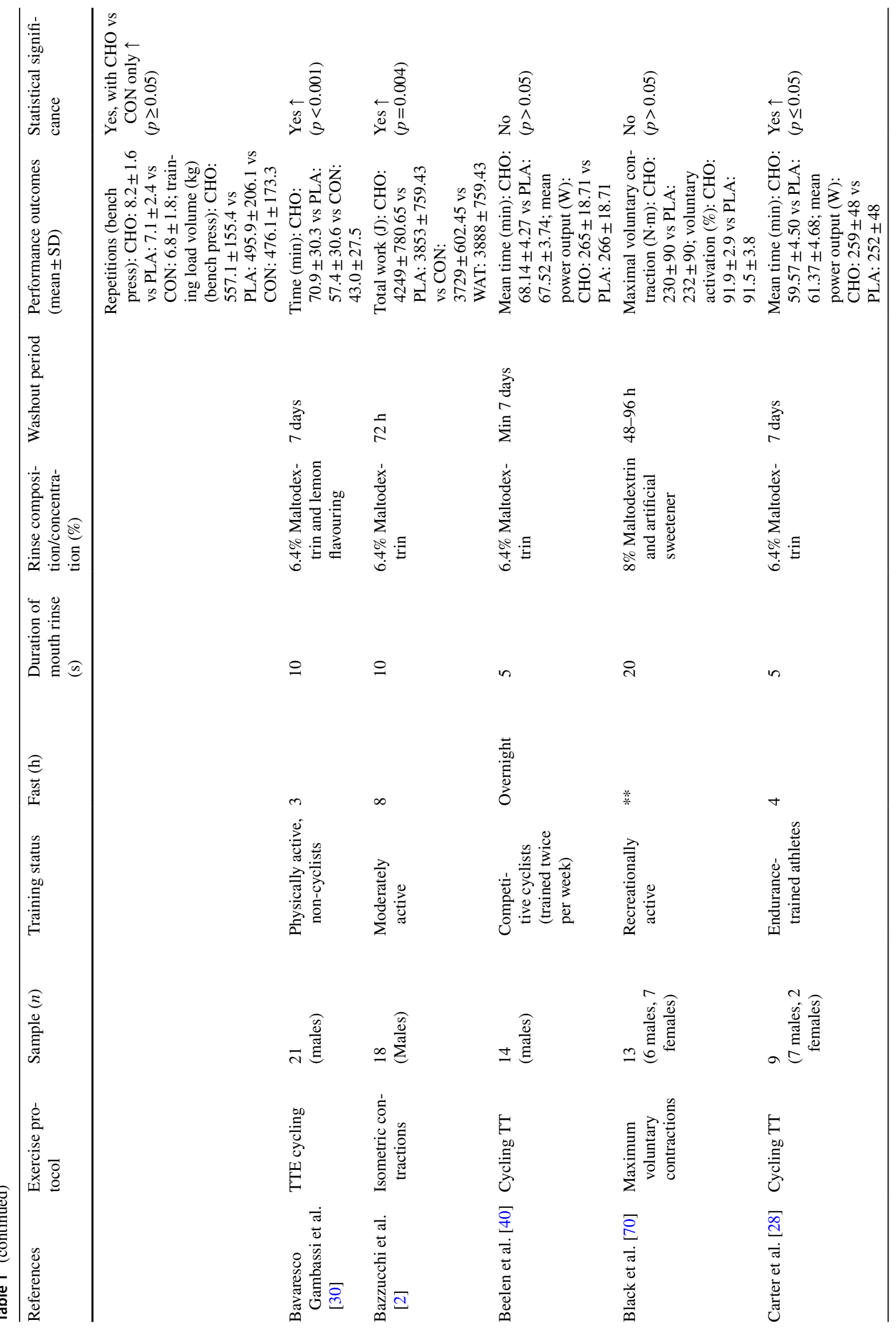




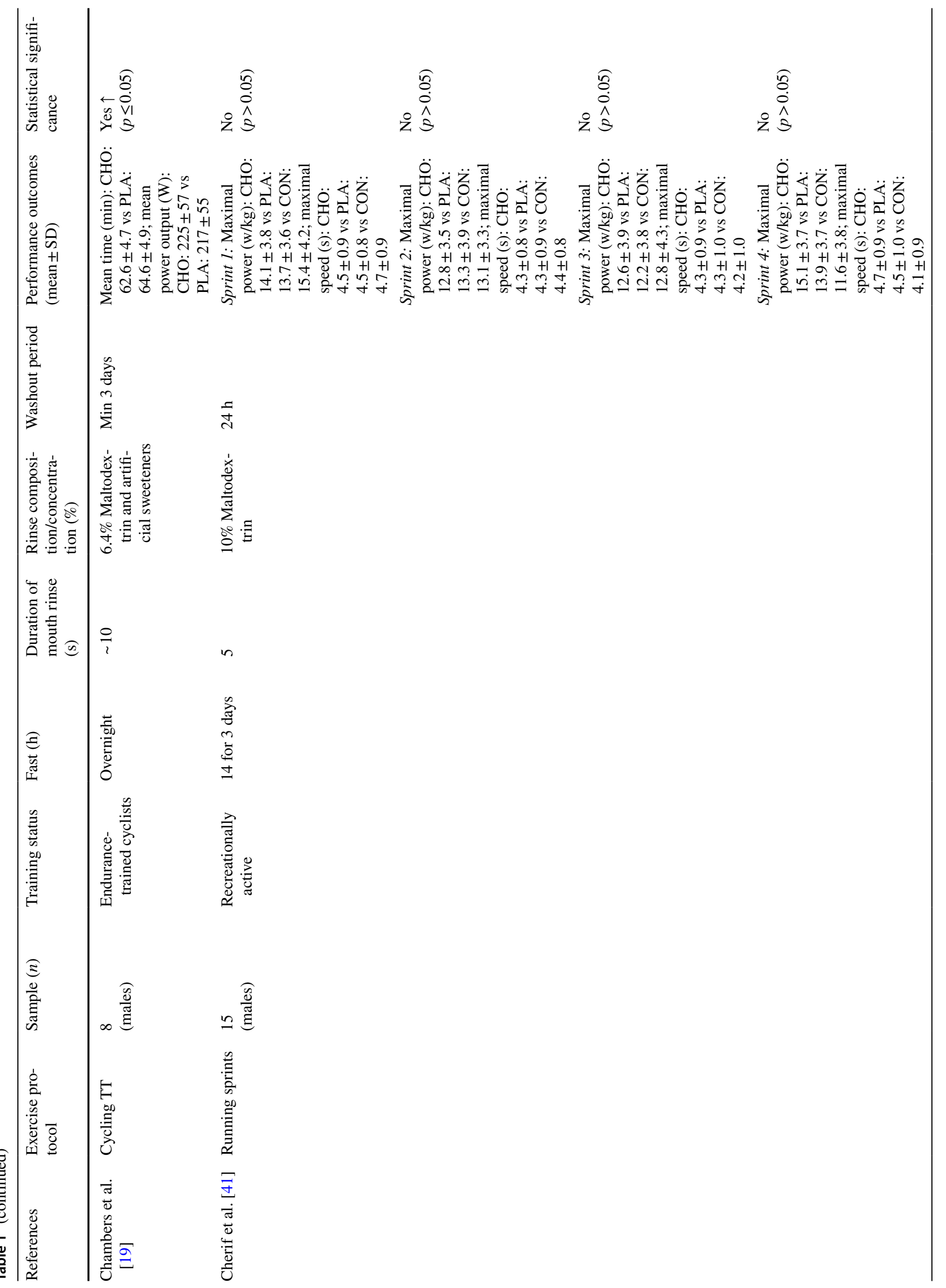


Carbohydrate Oral Rinsing and Exercise Performance: Systematic Review and Meta-Analysis

1841

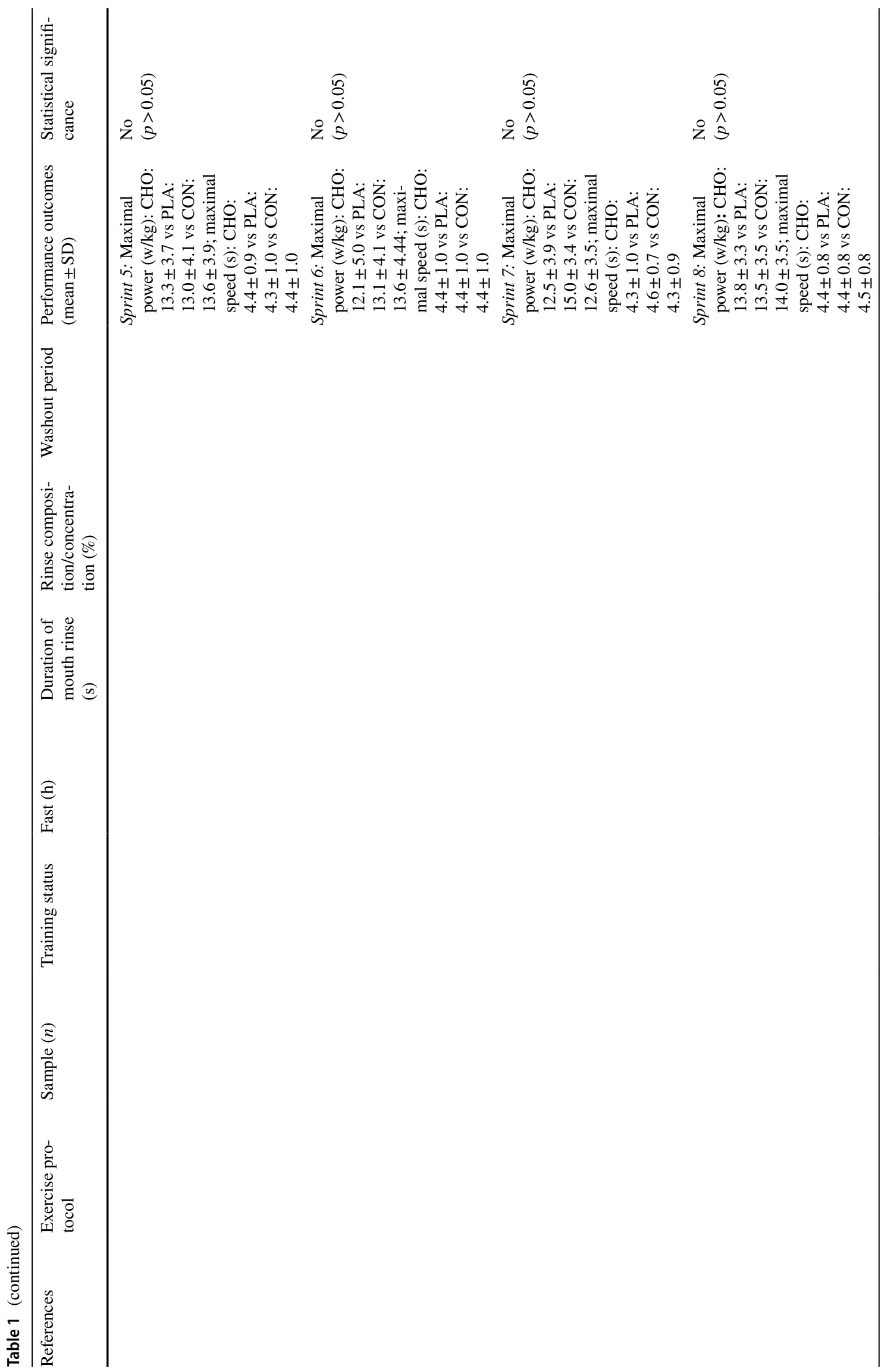




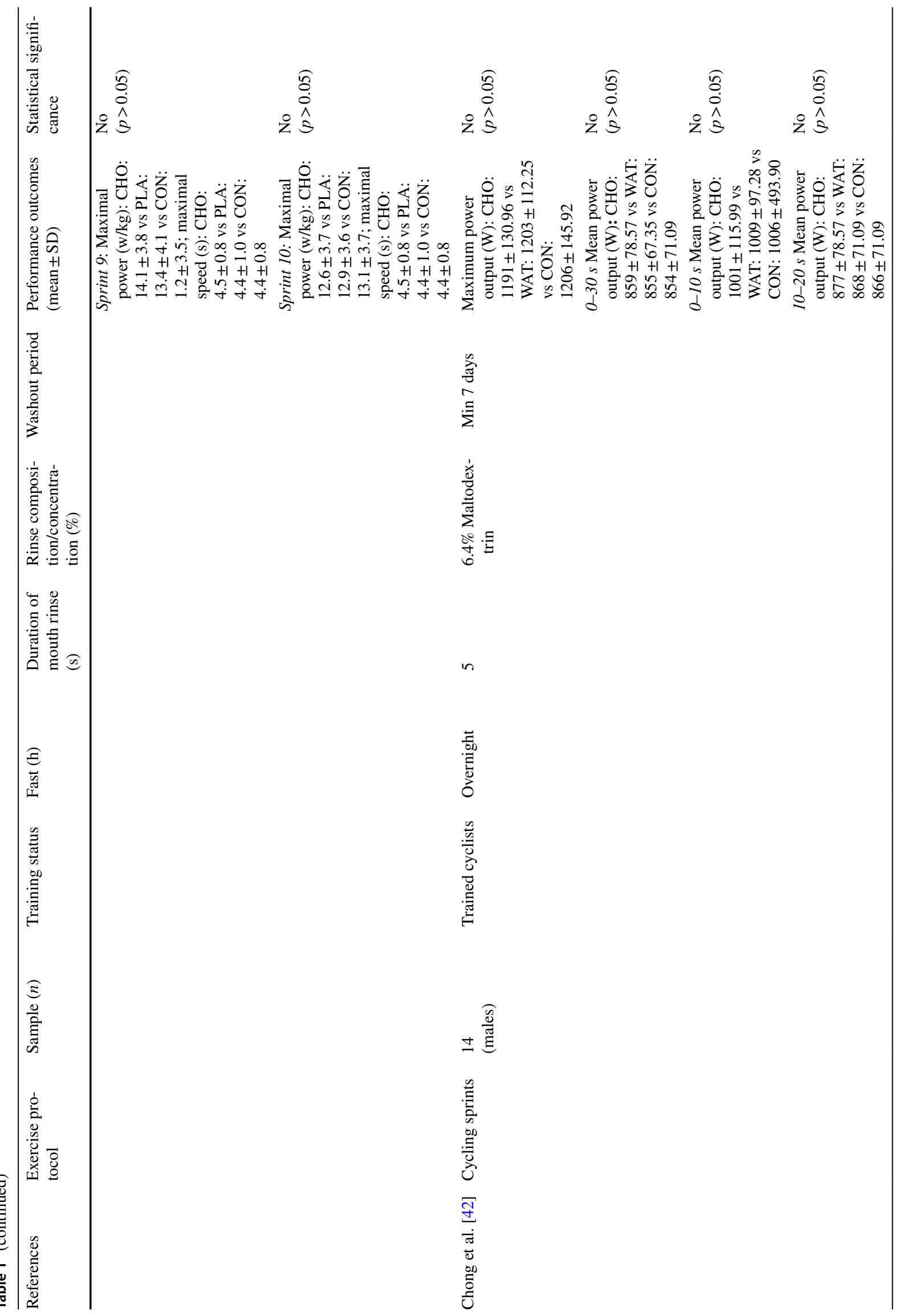




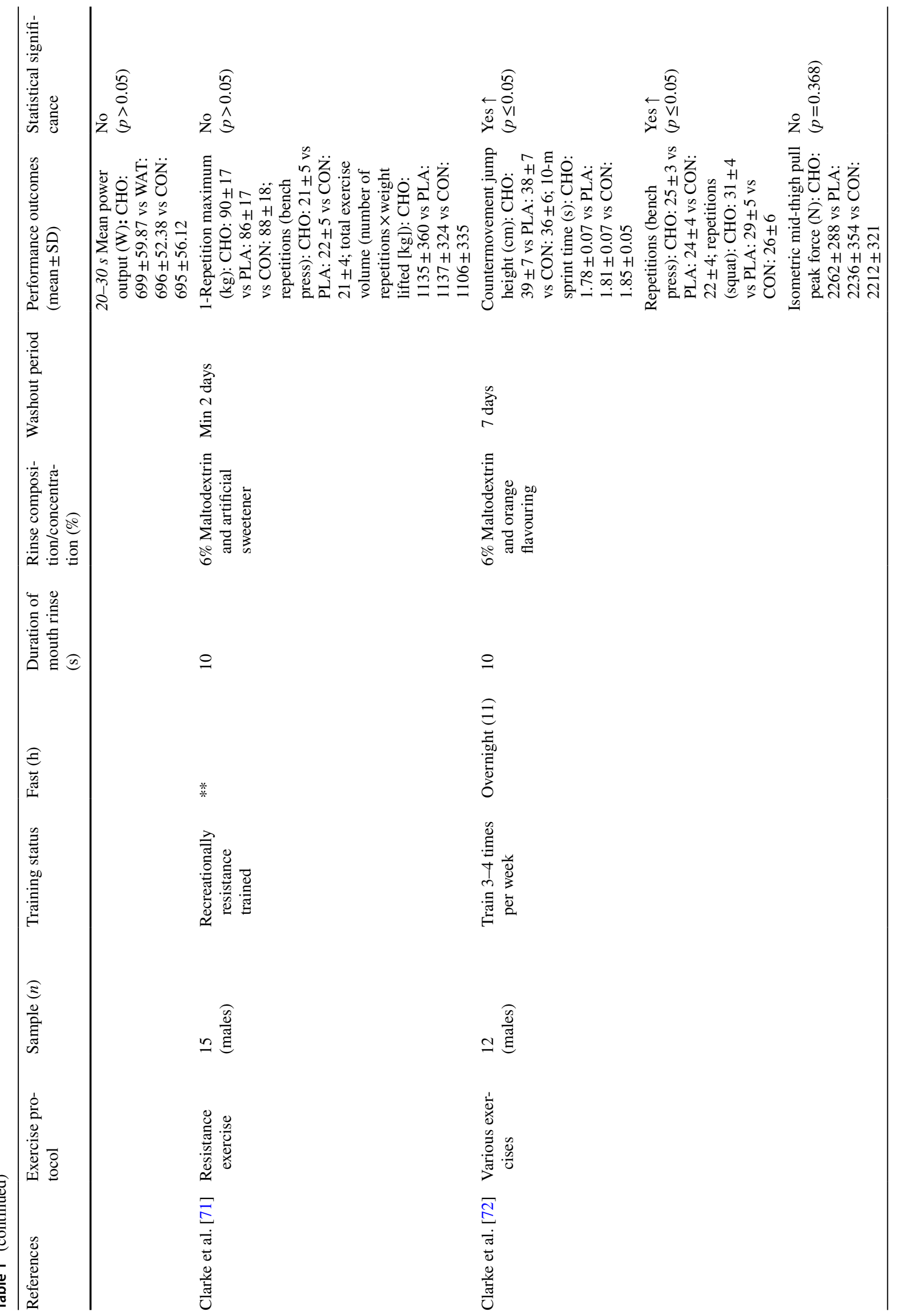




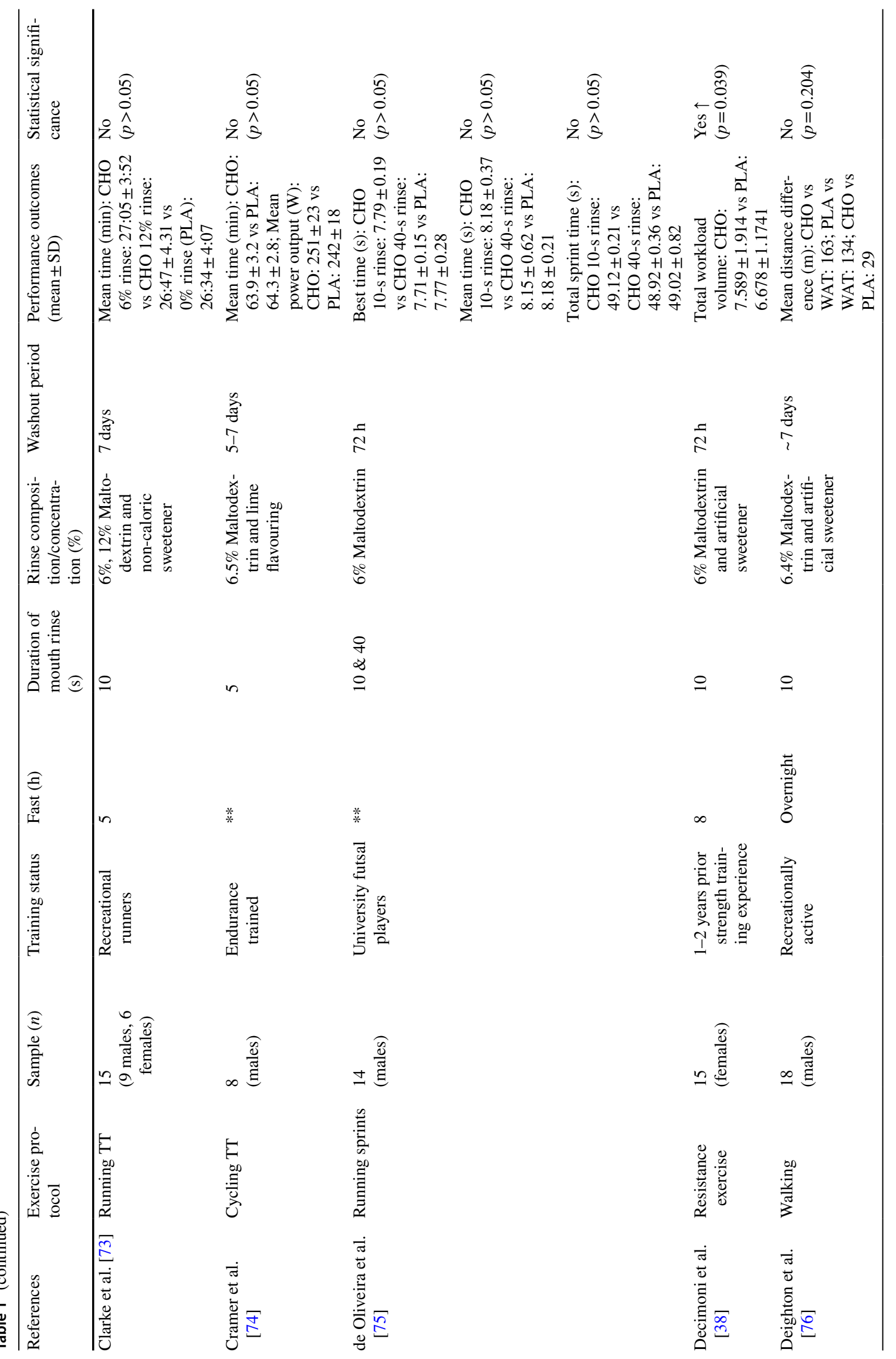




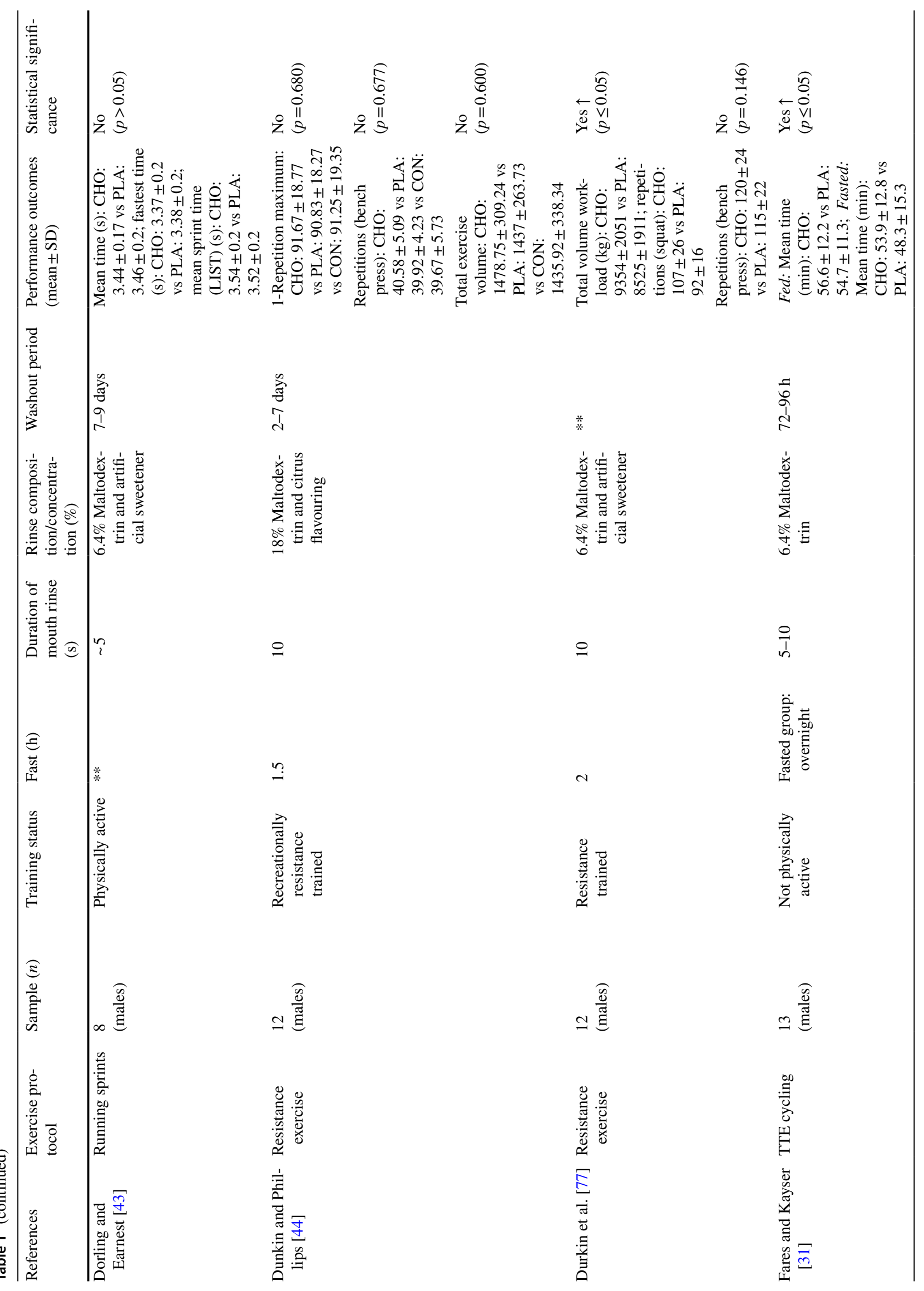




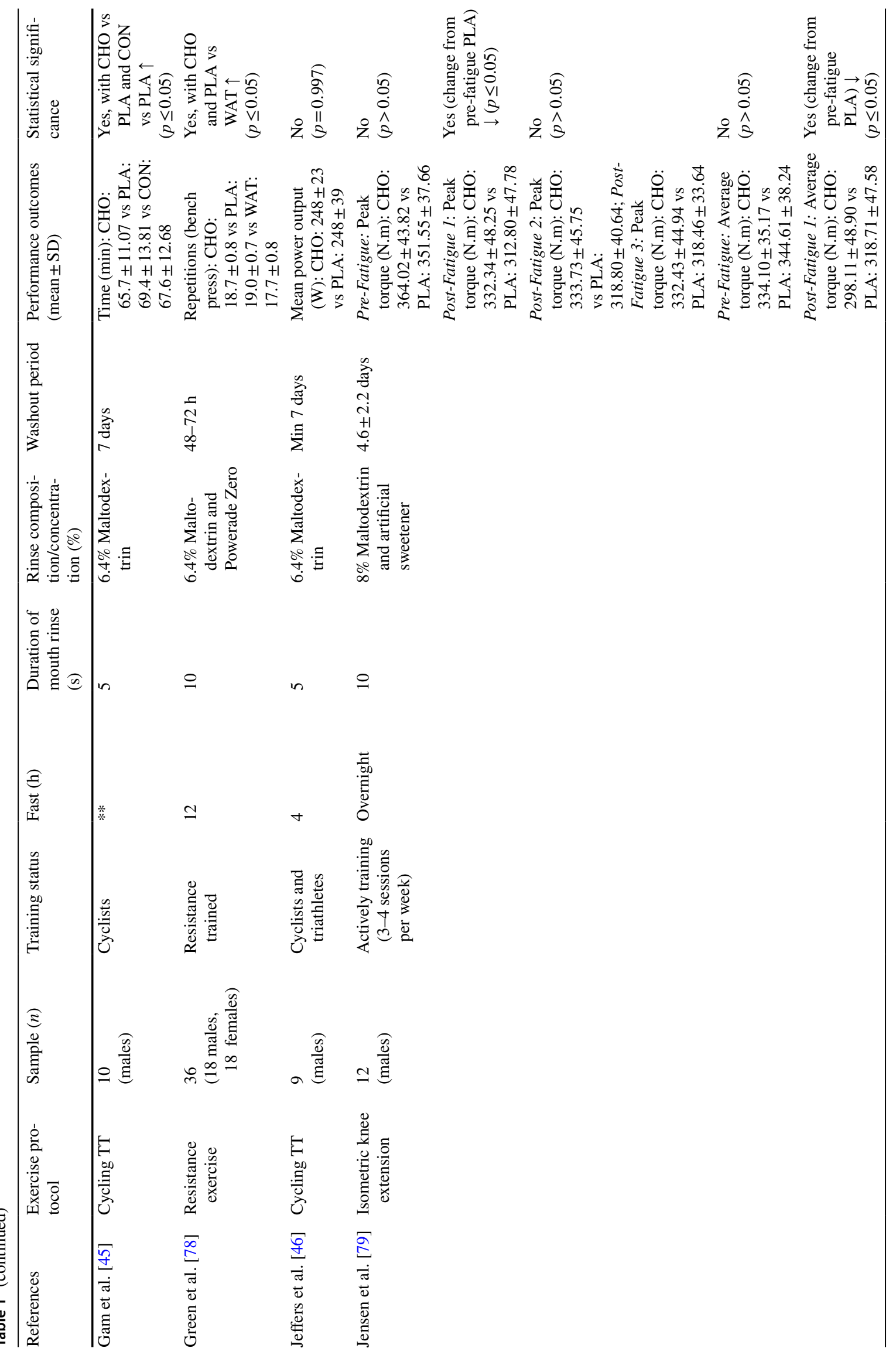


Carbohydrate Oral Rinsing and Exercise Performance: Systematic Review and Meta-Analysis

1847

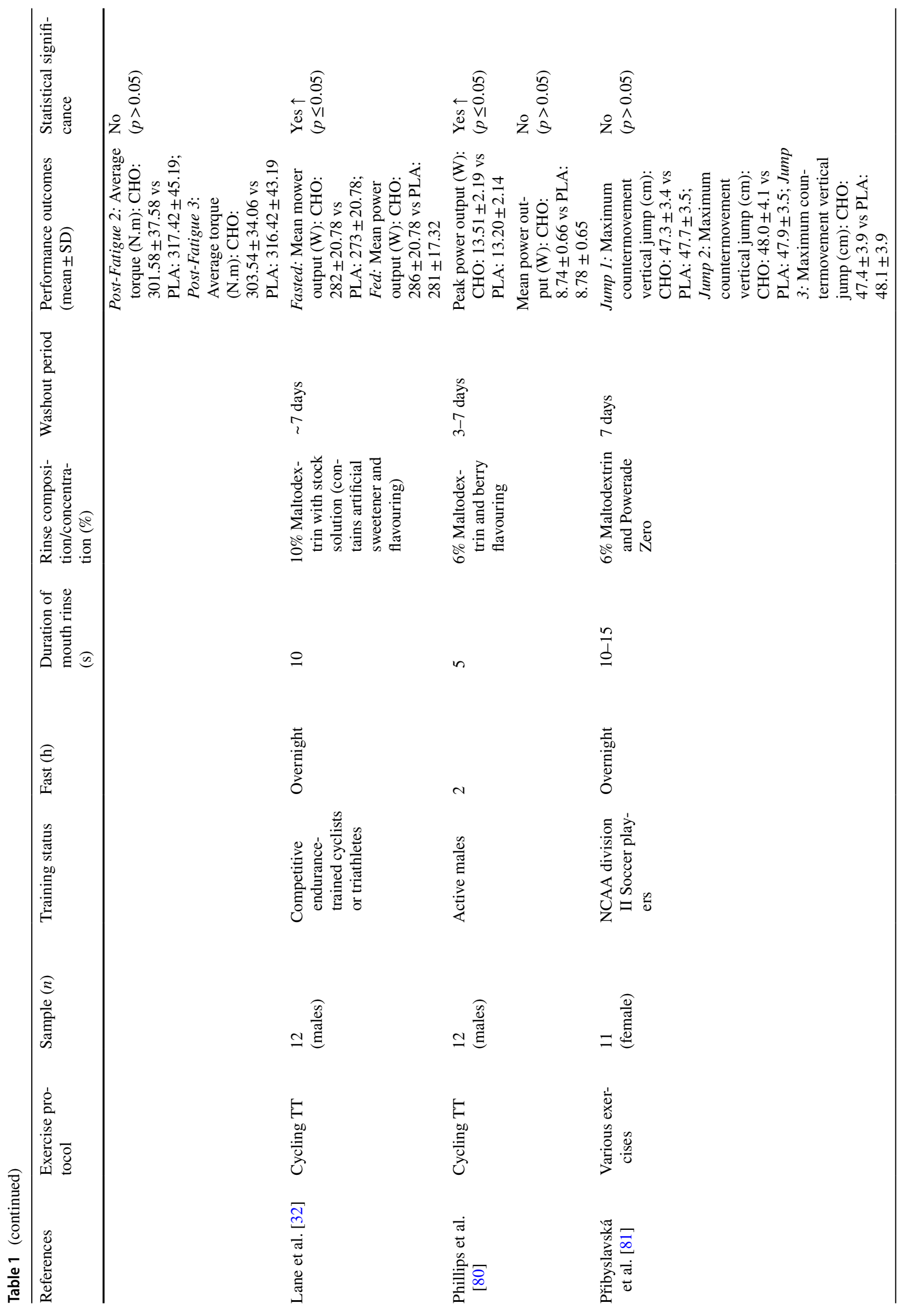




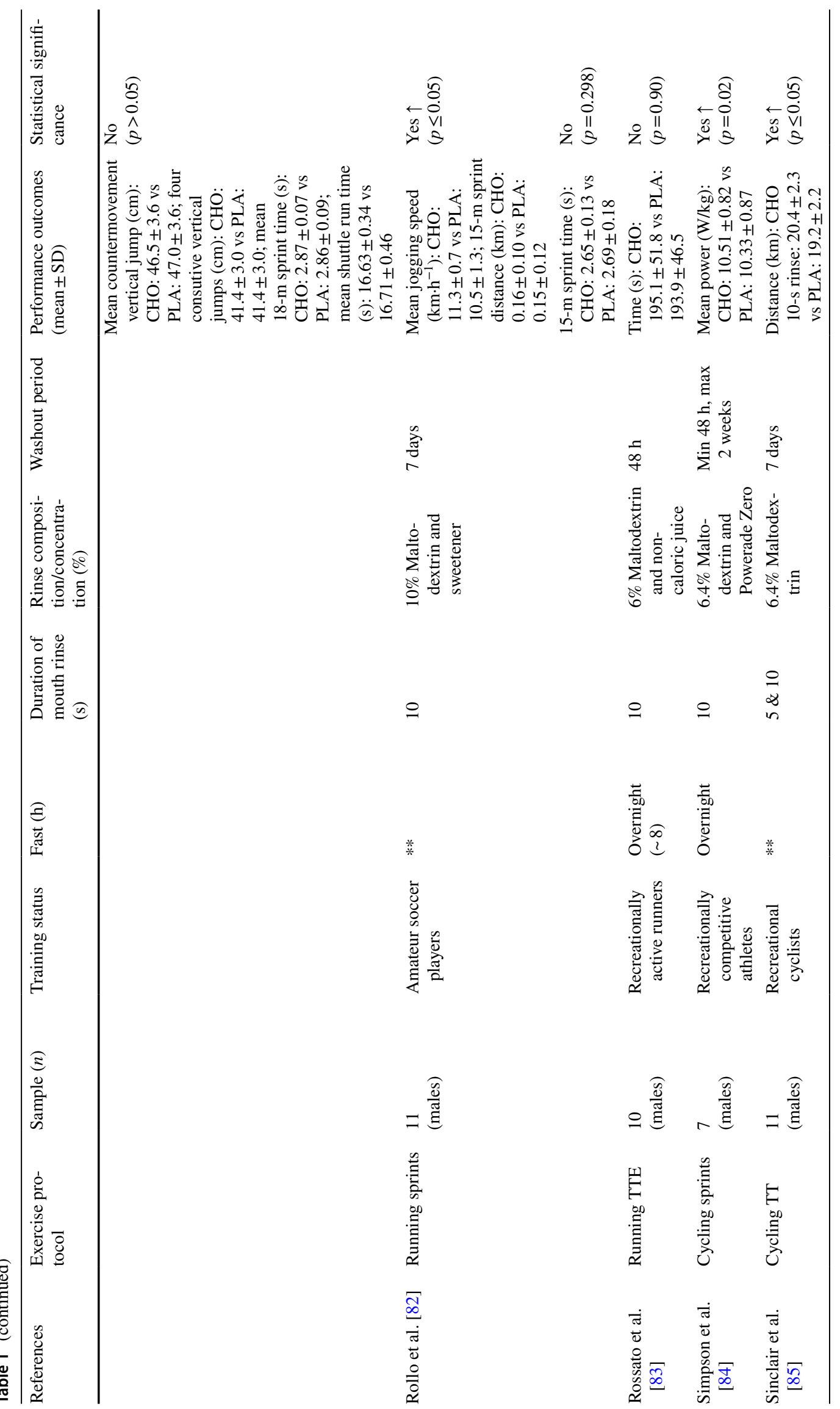




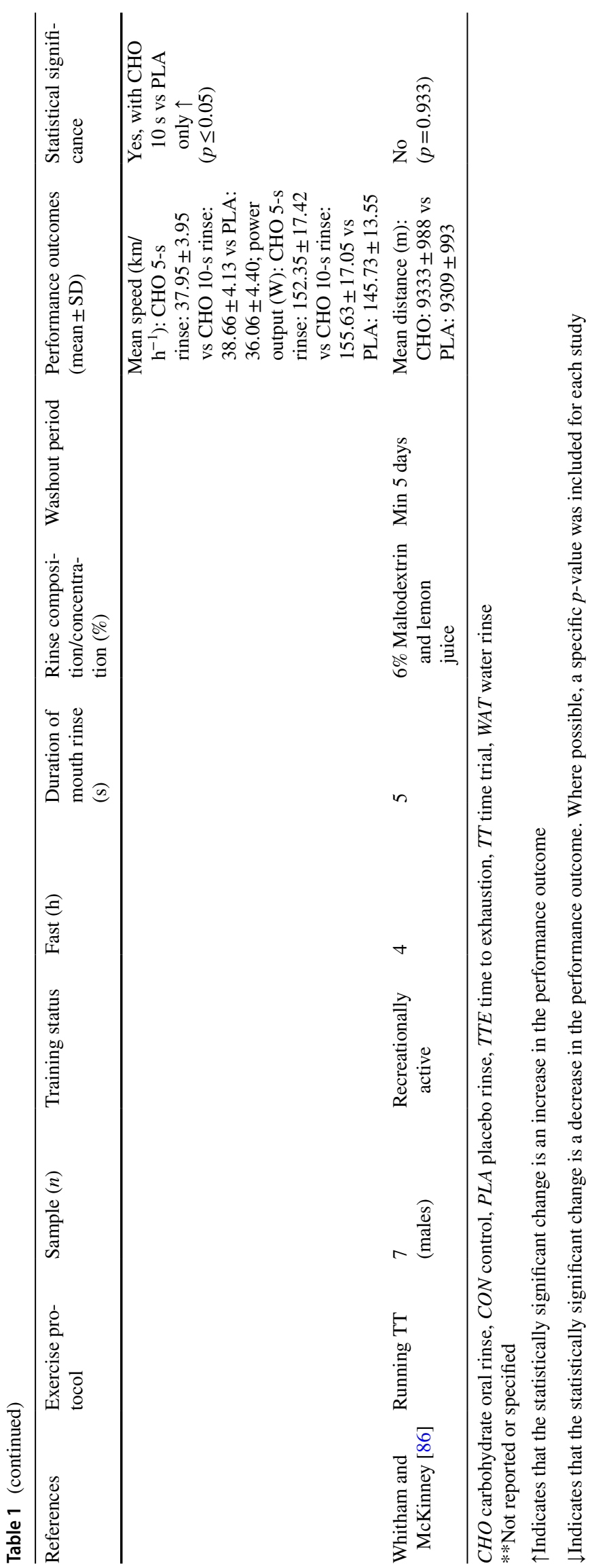


35 studies, the majority of included studies recruited only male participants $(n=28)$, while only two studies recruited female participants. The remaining studies recruited both males and females $(n=5)$.

\subsubsection{Oral Rinse Protocols}

Between the studies, there was a large variation in oral rinse protocol. The duration of rinsing ranged from five to $40 \mathrm{~s}$. From the total 35 studies, the majority of studies rinsed for either five $(n=12)$ or $10 \mathrm{~s}(n=20)$. The majority used a concentration of $6.4 \%(n=19)$; however, across the studies, the concentrations used varied between $6 \%(n=9), 6.5 \%(n=1)$, $8 \%(n=2), 10 \%(n=3), 12 \%(n=1)$ and $18 \%(n=1)$. Further information is shown in Table 1.

\subsubsection{Origins of Maltodextrin}

Twenty-two studies provided information on the type of maltodextrin or the manufacturer that was used in the research. Of all 35 studies, only a small number provided specific information on the composition of the maltodextrin $(n=3)$. Refer to Table 2 for a detailed list of this information. As highlighted previously, information concerning the origin or structure of the maltodextrin should be discussed. These are potentially confounding factors that could affect the efficacy of the carbohydrate oral rinse and any associated exercise performance improvements.

\subsubsection{Exercise Protocol}

The most common type of exercise protocol used in the included studies was a cycling time trial $(n=10)$. Time to exhaustion (TTE) cycling test protocol $(n=2)$ and a cycling sprinting protocol $(n=2)$ were also used. Multiple studies used protocols involving running-based sprinting $(n=4)$, while studies also used protocols involving a running time trial $(n=2)$ and a TTE running test $(n=1)$. The remaining studies included resistance exercise $(n=6)$, isometric contractions $(n=2)$, various exercises (i.e. 10-m running-based sprint, countermovement jump height, isometric mid-thigh pull peak force, repetitions [bench press and squat]) $(n=2)$, walking $(n=1)$, arm cranking $(n=1)$, isometric knee flexion $(n=1)$ and maximum voluntary contractions $(n=1)$.

\subsubsection{Performance Outcomes}

From the total of 35 studies that used a maltodextrin-based oral rinse, a proportion of these studies found a statistically significant improvement in exercise performance $(n=19)$, while the remaining studies found no significant improvements $(n=16)$. Across the studies, the most frequently reported performance outcomes were specific to
Table 2 Origins of maltodextrin used in the included studies

\begin{tabular}{|c|c|}
\hline Study & Origin of maltodextrin \\
\hline Andersson et al. [39] & MyProtein, Northwich, UK \\
\hline Ataide-Silva et al. [29] & Neonutri-Malto, $\mathrm{CHO}$ \\
\hline Bailey et al. [68] & NS \\
\hline Bastos-Silva et al. [69] & NS \\
\hline $\begin{array}{l}\text { Bavaresco Gambassi et al. } \\
\text { [30] }\end{array}$ & Athletica Nutrition, Matao, SP, Brazil \\
\hline Bazzucchi et al. [2] & NS \\
\hline Beelen et al. [40] & AVEBE (Veendam, The Netherlands) \\
\hline Black et al. [70] & L.D. Carlson Co., Kent, OH, USA \\
\hline Carter et al. [28] & NS \\
\hline Chambers et al. [19] & Roquette, France \\
\hline Cherif et al. [41] & SIS company, Nelson, UK \\
\hline Chong et al. [42] & $\begin{array}{l}\text { Polycose, Ross Laboratory, Columbus } \\
\text { OH }\end{array}$ \\
\hline Clarke et al. [71] & MyProtein, Manchester, UK \\
\hline Clarke et al. [72] & MyProtein, Manchester, UK \\
\hline Clarke et al. [73] & MyProtein, Cheshire, England, UK \\
\hline Cramer et al. [74] & NS \\
\hline de Oliveira et al. [75] & NS \\
\hline Decimoni et al. [38] & Body Action, Brazil \\
\hline Deighton et al. [76] & NS \\
\hline Dorling and Earnest [43] & HighFive, Bardon, England \\
\hline Dunkin and Phillips [44] & Bulk Powders TM, Colchester, UK \\
\hline Durkin et al. [77] & NS \\
\hline Fares and Kayser [31] & NS \\
\hline Gam et al. [45] & $\begin{array}{l}\text { Polycose, Ross Nutrition, Columbus, } \\
\text { OH }\end{array}$ \\
\hline Green et al. [78] & Natural Foods Inc., Toledo, $\mathrm{OH}$ \\
\hline Jeffers et al. [46] & NS \\
\hline Jensen et al. [79] & NS \\
\hline Lane et al. [32] & NS \\
\hline Phillips et al. [80] & HighFive, Bardon, Leicestershire \\
\hline Přibyslavská et al. [81] & Letco Medical, Decatur, AL \\
\hline Rollo et al. [82] & MuscleTalk, Northhamptonshire, UK \\
\hline Rossato et al. [83] & Health Labs, Belo Horizonte, Brazil \\
\hline Simpson et al. [84] & Home Brew Supply LLC, TX, USA \\
\hline Sinclair et al. [85] & NS \\
\hline Whitham and McKinney [86] & $\begin{array}{l}97 \% \text { polysaccharide, } 2 \% \text { disaccharide, } \\
1 \% \text { glucose; Roquette, Corby, UK }\end{array}$ \\
\hline
\end{tabular}

$N S$ not stated

time (including time to completion and time to exhaustion) $(n=21)$, power $(n=12)$, repetitions $(n=7)$, distance $(n=5)$ and countermovement jumps $(n=4)$. Additional performance outcomes included outcomes specific to speed $(n=3)$, maximal voluntary contractions $(n=3)$, total exercise volume $(n=3)$, work $(n=2)$, training load $(n=2)$, pull peak force $(n=1)$, motor evoked potential $(n=1)$ and torque $(n=1)$. Table 1 presents further information concerning 
performance outcomes, participant training status and exercise protocol.

\subsubsection{Fasting}

Of the 35 included studies, the majority of studies incorporated fasting into their protocol $(n=24)$ while the remaining studies did not specify if their participants exercised in a fed or fasted state $(n=11)$. Fasting times ranged from $90 \mathrm{~min}(n=1)$ to fasting overnight (approximately $8-12 \mathrm{~h}$ ) $(n=16)$. For a small portion of the studies, the fasting duration was $<5 \mathrm{~h}(n=8)$, while for the majority of studies, the fasting duration was $>5 \mathrm{~h}(n=16)$.

\subsubsection{Washout Period}

Of the 35 studies, the majority $(n=34)$ included information about a washout period in between trials. One study did not include information concerning washout periods and the author was contacted for further information, with no response received. The shortest reported washout period was $24 \mathrm{~h}(n=1)$ and the longest washout period was 7-9 days $(n=1)$. The most common washout period was 7 days $(n=9)$. Table 1 presents more detailed information.

\subsubsection{Quality Assessment}

All studies clearly reported the intervention and outcomes of their research. Of the 35 included studies, only eight sufficiently reported inclusion and exclusion criteria for their participant groups. Thirty-four studies reported participant demographic information and all studies $(n=35)$ included a representative population. The majority of studies $(n=34)$ sufficiently described the methods and protocol used throughout data collection. Twenty-three of the studies described the setting of data collection (e.g. a laboratorybased setting). Most studies disclosed information concerning randomisation $(n=28)$, double blinding $(n=24)$ or single blinding $(n=34)$ in their methodology. The majority of studies $(n=29)$ were based on valid research methods and referenced the original research. All studies $(n=35)$ used consistent measurements and discussed the findings of their research. None of the studies stated participant withdrawals hence no reasons for withdrawals were stated. Seventeen studies discussed biases or limitations of their research. Any potential conflicts of interest were disclosed by 19 of the studies and one paper partially did so. Results of the methodological quality assessment of the studies is presented in Online Resource 1 (see Electronic Supplementary Material [ESM]).

\subsection{Meta-Analysis Results}

The initial conventional pairwise meta-analysis results showed that the carbohydrate oral rinse increased exercise performance significantly when compared with the placebo rinse condition $(\mathrm{SMD}=0.15,95 \%$ CI $0.04,0.27 ; p=0.01$ ). However, from this analysis, moderate heterogeneity was observed $\left(I^{2}=20.70 \%, \mathrm{Tau}^{2}=0.04, \mathrm{Chi}^{2}=83.92, d f=57\right.$, $p=0.01)$ and as this approach assumes independence between each data point, this approach was not appropriate. To overcome this issue, a random effect robust meta-analysis model was used to compare the overall effect of carbohydrate rinsing on exercise performance. This more conservative adjusted model demonstrated that there was evidence of the carbohydrate oral rinse improving exercise performance in comparison with a placebo oral rinse $(\mathrm{SMD}=0.17,95 \%$ CI $-0.01,0.34 ; p=0.051)$. However, the model adjustment to the standard errors resulted in a wider, more conservative 95\% confidence interval and $p$-value (towards the null).

\subsection{Subgroup Analysis Results-Conventional Subgroup Meta-Analysis}

\subsubsection{Characterisation of Participants}

For the individual groups of male participants $(n=47)$ $(\mathrm{SMD}=0.11,95 \% \mathrm{CI}-0.004,0.23 ; p=0.058)$, female participants $(n=4)(\mathrm{SMD}=0.12,95 \% \mathrm{CI}-0.27,0.51$; $p=0.546)$ and combined (male and female participants) $(n=7)(\mathrm{SMD}=0.79,95 \% \mathrm{CI}-0.20,1.77 ; p=0.119)$, the mean effect size for these groups was not statistically significant at the 5\% level (Fig. 2). The combined group showed some unexplained between-study heterogeneity with estimated $I^{2}$ of $90.98 \%$ within this subgrouping.

\subsubsection{Oral Rinse Protocols-Rinsing Duration}

For the individual groups of rinsing for $5 \mathrm{~s}(n=17)$ (SMD $=$ $0.01,95 \% \mathrm{CI}-0.19,0.21 ; p=0.932), 7.5 \mathrm{~s}(n=1)$ (SMD $=0.28,95 \% \mathrm{CI}-0.47,1.03 ; p=0.461), 12.5 \mathrm{~s}(n=3)$ $(\mathrm{SMD}=-0.07,95 \% \mathrm{CI}-0.53,0.40 ; p=0.774), 20 \mathrm{~s}$ $(n=2)(\mathrm{SMD}=1.12,95 \% \mathrm{CI}-1.19,3.42 ; p=0.342)$ and $40 \mathrm{~s}(n=1)(\mathrm{SMD}=-0.06,95 \% \mathrm{CI}-0.78,0.66 ; p=0.864)$, the mean effect size was not statistically significant (Fig. 2). For the individual group of rinsing for $10 \mathrm{~s}(n=34)$ $(\mathrm{SMD}=0.22,95 \%$ CI $0.05,0.39 ; p=0.013)$, the mean effect size for this group was statistically significant at the $5 \%$ level. For this analysis, articles that provided a range for rinsing duration (e.g. 10-15 s), a middle point between the range was used for the analysis. Furthermore, for articles that provided an approximate rinsing time (i.e. $\sim 5 \mathrm{~s}$ ), a value of $4.9 \mathrm{~s}$ was used in the analysis. 
Fig. 2 Forest plot comparing the effects of the moderatorssex, rinse concentration (\%), rinse duration (s) and fastingon carbohydrate oral rinsing in comparison with a placebo condition. This forest plot was performed with a conventional random-effects REML (restricted maximum likelihood) model

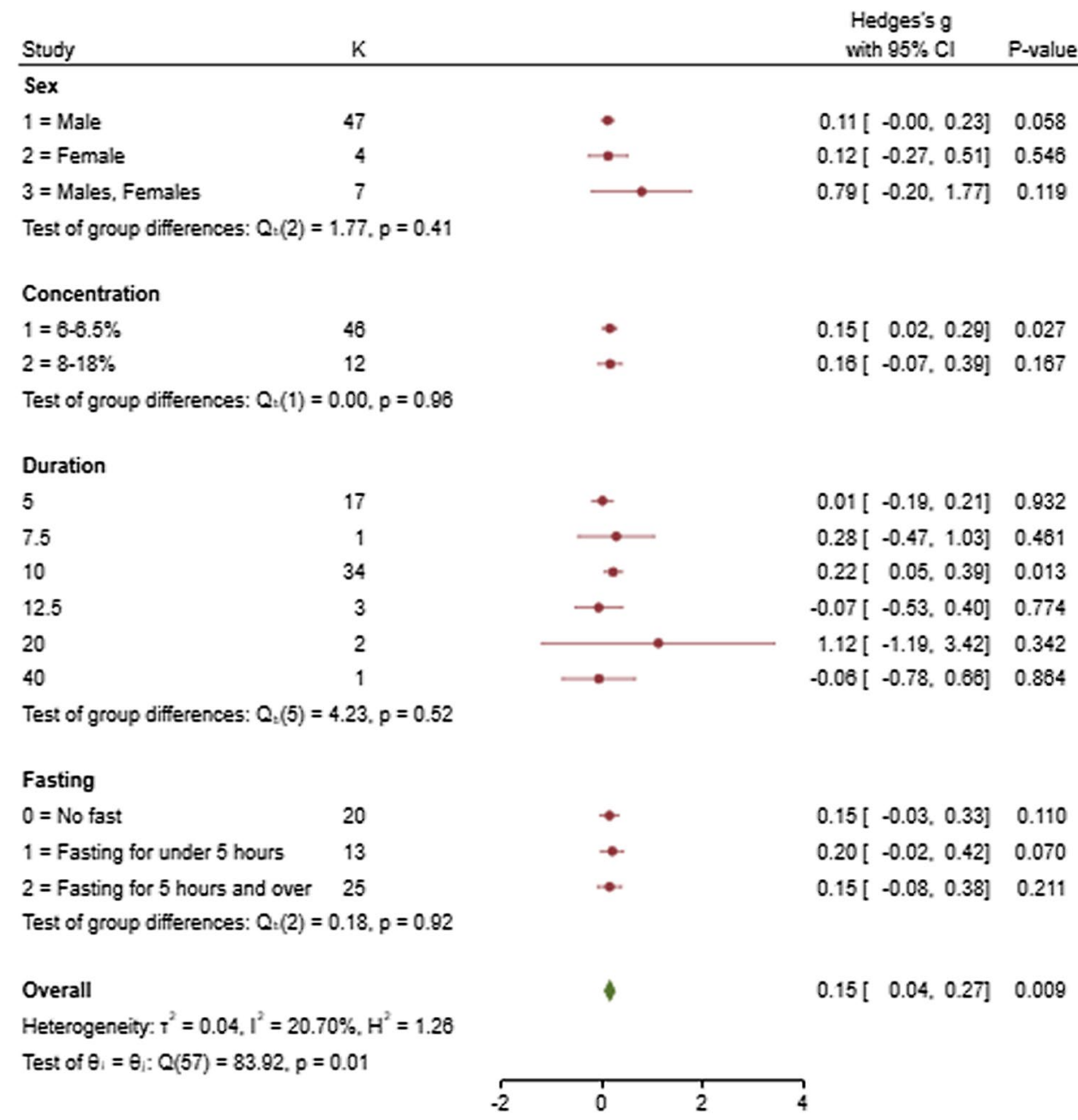

Random-effects REML model

\subsubsection{Oral Rinse Protocols-Rinse Concentration}

For the individual groups of rinse concentration of 6-6.5\% (group 1) and 8-18\% (group 2), the mean effect size for group $1(n=46)(\mathrm{SMD}=0.15,95 \% \mathrm{CI} 0.02,0.29 ; p=0.027)$ was statistically significant at the $5 \%$ level while the mean effect size for group $2(n=12)(\mathrm{SMD}=0.16,95 \%$ CI $-0.07,0.39 ; p=0.167$ ) was not statistically significant at the 5\% level (Fig. 2). The variable of ungrouped, individual rinse concentrations was examined in a continuous format which is available in Online Resource 2 (see ESM).

\subsubsection{Exercise Protocol}

For the individual groups of arm cranking $(n=1)(\mathrm{SMD}=$ $0.27,95 \% \mathrm{CI}-0.51,1.04)$, cycling $(n=18)(\mathrm{SMD}=0.07$, $95 \% \mathrm{CI}-0.13,0.26)$, isometric contractions $(n=4)$ (SMD $=0.58,95 \% \mathrm{CI}-0.49,1.67)$, resistance exercise $(n=15)$ $(\mathrm{SMD}=0.15,95 \% \mathrm{CI}-0.04,0.35)$, running $(n=17)$ $(\mathrm{SMD}=0.22,95 \% \mathrm{CI}-0.12,0.55)$, maximum voluntary contractions $(n=1)(\mathrm{SMD}=-0.02,95 \% \mathrm{CI}-0.77,0.72)$ and countermovement vertical jump $(n=2)(\mathrm{SMD}=0.01$, $95 \%$ CI $-0.55,0.57$ ), the mean effect size for these groups was not statistically significant at the 5\% level. The isometric contractions group and running group show some unexplained between-study heterogeneity with estimated $I^{2}$ of $86.16 \%$ and $64.84 \%$, respectively.

\subsubsection{Fasting}

For the individual groups of no fasting $(n=20)$ (SMD $=0.15,95 \% \mathrm{CI}-0.03,0.33 ; p=0.110)$, fasting for $<5 \mathrm{~h}$ $(n=13)(\mathrm{SMD}=0.20,95 \% \mathrm{CI}-0.02,0.42 ; p=0.070)$ and fasting for $>5 \mathrm{~h}(n=25)(\mathrm{SMD}=0.15,95 \% \mathrm{CI}-0.08,0.38$; $p=0.211$ ), the mean effect size for these groups was not statistically significant at the 5\% level (Fig. 2). The group of fasting for $>5 \mathrm{~h}$ shows some unexplained between-study heterogeneity with estimated $\mathrm{I}^{2}$ of $71.05 \%$. 


\subsection{Subgroup Analysis Results-Meta-Regression Model with Robust Variance Estimation}

\subsubsection{Characterisation of Participants}

There was no significant difference at the 5\% level when comparing the individual group of male participants with female participants (difference between SMDs $=0.17,95 \%$ $\mathrm{CI}-2.75,3.08 ; p=0.68$ ) or the combination of male and female participants (difference between SMDs $=0.42,95 \%$ CI $-0.98,1.82 ; p=0.48)$. All data points $(n=58)$ were included in this analysis.

\subsubsection{Oral Rinse Protocols-Rinsing Duration}

There was no significant difference at the 5\% level when comparing the individual group of rinsing for $5 \mathrm{~s}$ with rinsing for $10 \mathrm{~s}$ (difference between SMDs $=0.19,95 \%$ CI $-0.07,0.45 ; p=0.15)$. In a sensitivity analysis, only 51 data points were included in the meta-regression analysis as small sample adjustments could not be done with groups with fewer than four.

\subsubsection{Oral Rinse Protocols-Rinse Concentration}

There was no significant difference at the $5 \%$ level with group 1 (6-6.5\%) compared with group 2 (8-18\%) (difference between SMDs $=-0.07,95 \%$ CI $-0.36,0.22$; $p=0.58$ ). All data points were included in this analysis.

\subsubsection{Exercise Protocol}

There was no significant difference at the $5 \%$ level when comparing the individual group of cycling with isometric contractions (difference between SMDs $=0.52,95 \%$ CI $-1.15,2.19 ; p=0.42$ ), resistance exercise (difference between SMDs $=0.09,95 \% \mathrm{CI}-0.30,0.48 ; p=0.62$ ) or running (difference between SMDs $=0.17,95 \%$ CI $-0.35,0.69$; $p=0.49$ ). In a sensitivity analysis, only 54 data points were included in the meta-regression analysis as small sample adjustments could not be done with groups with fewer than four.

\subsubsection{Fasting}

There was no significant difference at the $5 \%$ level when comparing the individual group of no fasting with fasting group $1(<5 \mathrm{~h})$ (difference between SMDs $=-0.04,95 \%$ $\mathrm{CI}-0.42,0.33 ; p=0.81)$ or fasting group $2(>5 \mathrm{~h})$ (difference between SMDs $=-0.11,95 \% \mathrm{CI}-0.55,0.34$; $p=0.62$ ). All data points were included in this analysis.

\subsection{Risk of Bias Assessment Results}

Overall, of the 34 total studies (58 data points) included in this meta-analysis, all demonstrated a high level of evidence. The majority of studies reported using random sequence generation $(n=28)$ and all studies scored a low risk of bias in the categories of incomplete data $(n=34)$ and selective reporting $(n=34)$. A proportion of studies had a singleblinded study design $(n=10)$ and therefore scored an unclear risk of bias for that category. Furthermore, for the category of detection bias, all studies $(n=34)$ scored an unclear risk of bias. Results of the risk of bias assessment of the studies is presented in Online Resource 3 (see ESM).

\section{Discussion}

The purpose of this systematic review and meta-analysis was to investigate the published data examining the effects of a maltodextrin-based, carbohydrate oral rinse on exercise performance. This is the first review to examine the effects of a maltodextrin-based oral rinse on exercise performance and additionally to account for the issue of multiple data points from selected studies when performing the analysis. The key findings from this review were that evidence from the adjusted, random effects meta-regression approach suggests that a maltodextrin-based, carbohydrate oral rinse may cause improvements in exercise performance. Although the conventional meta-analytic approach demonstrated that oral rinsing with a 6-6.5\% maltodextrin-based, carbohydrate oral rinse for $10 \mathrm{~s}$ was the most effective at improving exercise performance, this approach was deemed to be inaccurate.

The systematic review and meta-analysis published by Brietzke et al. [49] examined the effects of carbohydrate rinsing on cycling time trial performance. Brietzke et al. [49] found that during a cycling time trial, a carbohydrate oral rinse improved mean power output but not the time to completion. However, a limitation exists with the meta-analyses that were performed in this review. In these analyses, multiple data points from three separate studies were included and this was not considered when performing a standard random effects model for the analysis. Similarly, the systematic review and meta-analysis from Pochmuller et al. [51] examined the effects of carbohydrate supplementation on performance trials. Pochmuller et al. [51] found that for trained male cyclists, there may be benefits to ingesting carbohydrates in a concentration of 6-8\% prior to and/or during exercise longer than $90 \mathrm{~min}$. To yield more homogeneous study designs, the data was divided into four groups for analysis. However, within these groups, multiple data points from studies were used in the analysis, which was not taken into account or adjusted for. Subsequently, this may have resulted in the analyses being inaccurate or too imprecise. 


\subsection{Characterisation of Participants}

According to the conventional meta-analytic method, there is evidence that the moderator of sex contributed to an improvement in exercise performance. Of the groups in the meta-analysis, only the male participant group was close to reaching significance. The results may be due to confounding factors that were independent of carbohydrate oral rinsing. Differences between males and female participants were not accounted for. However, according to the adjusted, conservative, meta-regression model using robust variance estimation, there was no indication that the moderator of sex contributes to an improvement in exercise performance with carbohydrate oral rinsing. While the majority of studies in this area recruit male participants, they are often classified as physically active or participate in moderate to high levels of activity [2, 29, 30, 39, 40, 43]. Additional research has recruited participants that either train competitively or are experienced in that particular mode of exercise (i.e. running and cycling) $[32,40,42,45,46,73]$ and this is a potential reason for variation in the classification of participants recruited in the studies. There are several advantages of recruiting a specific sample group such as trained or experienced runners for exercise testing such as a running time trial. One advantage is that the risk of injury for the participant is lower. For example, novice runners have the highest proportion of injury in comparison with more experienced runners [87-89]. Additionally, trained participants may provide more consistent data as they require fewer familiarisation sessions prior to testing to decrease possible learning effects and to achieve a higher level of reproducibility [90]. This is particularly imperative with exercise tests such as a cycling time trial where self-pacing during the trial is necessary.

\subsection{Oral Rinse Protocols-Rinsing Duration}

As demonstrated using the conventional meta-analytic method, of the reported rinsing conditions, exposure of the carbohydrate-based oral rinse in the oral cavity for $10 \mathrm{~s}$ was statistically significant in improving exercise performance. Conversely, according to the adjusted, conservative, metaregression model using robust variance estimation, duration of exposure of the rinse in the oral cavity did not significantly improve exercise performance.

The results from the conventional meta-analytic approach is consistent with research undertaken by Sinclair et al. [85] where the results of a 5-s and a 10-s rinse on exercise performance were compared. The authors reported that participants cycled further in the trial with a 10-s rinse in comparison with the 5 -s rinse trial. These findings suggest a duration dose response for the carbohydrate oral rinse. Studies that used longer rinse times of $20 \mathrm{~s}$ [68] and $60 \mathrm{~s}$
[91] did find improvements in exercise performance compared with a placebo rinse. However, it has been suggested that longer rinse times may cause loss of attention and focus while exercising, which can result in transient declines in power output [45] and can also affect performance through the impairment of breathing entrainment, which is defined as the rhythm of breathing synchronised with the rhythm of exercise [45]. Such a response could cause a reduction of oxygen uptake for a certain workload [92, 93] and also may allow for cyclists to reduce the energy costs of exercise [93]. A limitation for this current conventional meta-analysis approach was that the groups of $7.5 \mathrm{~s}, 20 \mathrm{~s}$ and $40 \mathrm{~s}$ had two or fewer studies in the subgroup analyses, which can underpower the analysis.

\subsection{Oral Rinse Protocols-Rinse Concentration}

The results from the conventional meta-analysis show that when the data was divided into two groups $(6-6.5 \%$ and $8-18 \%)$, of the two groups, only the concentration range of $6-6.5 \%$ was found to be statistically significant. It is possible that as the concentration of the rinses increases, it reaches a point where little to no improvements to exercise performance are evident, given that in studies using higher concentrations (10\% [41], 12\% [73] and 18\% [44]), no improvements in exercise performance were evident. Further, James et al. [21] reported performance improvements with a $7 \%$ rinse; however, these results did not increase when the concentration of the rinse was increased to $14 \%$. However, according to the meta-regression model using robust variance estimation, the moderator of rinse concentration did not significantly improve exercise performance. These inconsistent findings suggest that perhaps a combination of factors (not purely rinse time or rinse concentration) may elicit a performance improvement in exercise. In contrast, research by Jensen et al. [79], Lane et al. [32] and Rollo et al. [82] used $8 \%, 10 \%$ and $10 \%$ rinses, respectively, and did find significant improvements in exercise performance with a carbohydrate oral rinse in comparison with a placebo rinse. A limitation for this current conventional meta-analysis approach was that the group of $12 \%$ rinse concentration had one study in the subgroup analysis, which can underpower the analysis.

\subsection{Exercise Protocol}

The results from the conventional meta-analytic approach demonstrate that the moderator of exercise protocol did not significantly contribute to an exercise performance improvement with a carbohydrate oral rinse. However, the specific exercise protocol of cycling, resistance exercise and running demonstrated some evidence of a performance improvement and the inclusion of further original, independent studies in 
the current analysis may have resulted in a significant finding. Furthermore, according to the adjusted, conservative, meta-regression model using robust variance estimation, the moderator of exercise protocol did not significantly impact exercise performance improvement with a carbohydrate oral rinse.

Across the literature, improvements in both power output and performance time, associated with a carbohydrate oral rinse, have been reported in various forms of exercise including cycling [19, 28-33]. In cycling-based studies, time trials $[19,29,32,40,45,46,80]$, sprints $[42,84]$ and time to exhaustion tests $[30,31]$ on a stationary cycle ergometer were used. A potential explanation for the observed improvements in cycling performance is the inclusion of familiarisation sessions in some studies prior to commencing experimental testing $[19,28-30,32,40,42,45,80,84$, 85 ]. The inclusion of familiarisation trials can increase reliability and reduce the likelihood of learning effects [90], implications that are particularly relevant to exercise tests that require self-pacing by participants (e.g. time trials). Across the studies included in this review, a small selection of studies did not include information on familiarisation protocol $[31,38,46,69,74,77]$. It is currently recommended that for experienced participants, at least one familiarisation trial is required for reproducibility of performance [94-97] and to establish a stable pacing strategy [98].

Similarly, exercise performance improvements with carbohydrate oral rinsing have also been evident in running [34-37]. In running-based studies, the mode of exercise included sprints $[41,43,82]$ and time trials $[73,86]$. Unlike cycling-based exercise tests, where tests are typically conducted on a cycle ergometer, research methods in runningbased studies were mixed, using stationary treadmill running $[41,83,86]$ and self-paced running indoors $[43,72,75,81$, 82] and outdoors [73]. Such variation in exercise protocols can affect overall validity and reliability, and increase variability [99]. For example, laboratory-based running protocols typically have a decreased level of ecological validity [100]. In comparison with self-paced running outside, the decision to consciously control the speed of the treadmill does not occur as often or as swiftly [101, 102]. Small but significant differences also exist between running on a treadmill and running on a track including variation in movement patterns, ground surface and airstream [103].

Improvements in exercise performance associated with carbohydrate oral rinsing have also been found in resistance exercise protocols [38, 77, 78]. In resistance exercise-based studies, the specific exercises included leg press, bench press, military press, seated row and half-squat [38] and bench press protocols $[44,78]$. Oral rinsing with maltodextrin has also resulted in improvements in total workload volume during resistance-based exercises in comparison with a placebo $[38,77]$. However, this is in comparison with other research using resistance-based exercise where no performance improvements were evident with a carbohydrate oral rinse [44].

Additionally, improvements in exercise performance associated with carbohydrate oral rinsing have also been demonstrated in protocols incorporating isometric contractions [68] and isometric knee extension [79]. Using isometric knee extension, Jensen et al. [79] reported a significant decline in torque with a maltodextrin oral rinse in comparison with a placebo. Bailey et al. [68] demonstrated increases in maximal voluntary contractions with a maltodextrin oral rinse in comparison with a placebo. However, a limitation across the existing literature is that the methods used do not quantify effects on functional activities (e.g. squatting) [68]. Functional activities and training are more representative of daily movement and general exercise movement patterns [104]. One previous study used an arm cranking protocol [39]. However, this study did not find significant differences in distance $(\mathrm{km})$ covered during a 30 -min trial with a maltodextrin rinse when compared with a placebo. Furthermore, the aforementioned studies that use resistance exercise, arm cranking, isometric flexion, contraction and extension as modes of exercise to examine the effects of a carbohydrate oral rinse on exercise performance have limited applicability to sport performance more broadly, or specific exercise classifications (e.g. endurance or sprint-based exercise). A limitation for the current conventional meta-analysis approach was that the groups of arm cranking, maximum voluntary contractions and countermovement vertical jump had two or fewer studies in the analysis, which can result in an underpowered analysis for that group.

\subsection{Fasting}

From the results from the conventional meta-analytic method, it was unclear as to the effect of fasting on exercise performance with a carbohydrate oral rinse. All groups were close to reaching significance. Furthermore, according to the meta-regression model using robust variance estimation, there were no significant effects of fasting on exercise performance while using a carbohydrate oral rinse. These unclear findings are reflected in the literature as it has not been conclusively determined whether exercising in the preprandial or postprandial state contributes to a performance improvement. Fares and Kayser reported improvements in cycling performance time with a maltodextrin-based oral rinse in comparison with a placebo in both preprandial (overnight fasting) and postprandial states [31]. However, this finding of exercise improvements in both the preprandial and prandial state is in contrast with a large amount of literature in the area. For example, Lane et al. [32] found greater power 
output during a cycling time trial with a maltodextrin oral rinse (in comparison with a placebo) in a fasted state compared with a fed state $(3.4 \%$ vs $1.8 \%$ performance enhancement, respectively). Exercising in a fasted state could possibly be necessary for a carbohydrate-based oral rinse to improve exercise performance. These possible benefits of the carbohydrate oral rinse may therefore be in part dependant on endogenous carbohydrate (liver and glycogen) stores [40]. However, other studies have reported no performance improvements in a fed state in comparison with a fasted state [40, 86]. Beelen et al. [40] reported no improvements in performance time during a cycling time trial in a fed state in comparison with a fasted state and Whitham and McKinney [86] also found no increases in distance ran during a 45-min trial in a fed state in comparison with a fasted state while using a maltodextrin oral rinse in comparison with a placebo. Future research should aim to further investigate the effects of fasting on exercise performance while using a carbohydrate oral rinse.

\subsection{Types of Maltodextrin}

A limitation of the current research in the area is that the type of maltodextrin each study used in the rinse is often not specified. While most studies do include the manufacturer information of the maltodextrin, few studies include specific information on the composition of the maltodextrin [42, 45, 86]. Two of the studies included in the current review used Polycose (a maltodextrin with a composition of $91 \%$ glucose oligomers and polymers, $7 \%$ maltose and $2 \%$ glucose [105]) $[42,45,63]$ and one study used a maltodextrin with a $97 \%$ polysaccharide, $2 \%$ disaccharide and $1 \%$ glucose composition [86]. When such information is provided, further observations can be made which might facilitate further understanding as to how carbohydrate oral rinses improve exercise performance. Without information on the maltodextrin origin (e.g. corn, rice, manioc, oat or potato starch) and structure (amylose and amylopectin ratio, DE and DP), it becomes difficult to make direct comparisons on the performance improvements between studies as the various types of maltodextrin may affect the overall effect of the carbohydrate oral rinse and, in turn, perhaps the findings and results from each study. Future research should aim to specify the composition of the maltodextrins (including information on both DE and origin). Furthermore, more research may be necessary to identify the optimal composition of maltodextrins to trigger the exercise performance increase when using a carbohydrate oral rinse.

\subsection{Limitations of Carbohydrate Oral Rinsing}

One possible limitation of carbohydrate oral rinsing is the risk of choking on the rinse during exercise; however, no published research has discussed this risk. Furthermore, research by Gam et al. [45] showed that the action of mouth rinsing itself in a cycling time trial may result in impaired exercise performance. Their findings show in a cycling time trial that a water-based placebo rinse resulted in a $2.7 \%$ increase in completion time compared with the no-rinse control. They hypothesise that rinsing may cause a loss of attention and ability to focus on the task while also impairing breathing entrainment, which therefore resulted in transient declines in power output [45]. However, the addition of carbohydrates to the mouth rinse was found to oppose this decrease in performance associated with rinsing and may provide additional benefits as opposed to not rinsing at all [45]. These findings contrast, however, with another study that reported similar results with a water-based placebo rinse and a no-rinse control [106]. This study by Gam et al. [45] is the first to discuss limitations associated with carbohydrate oral rinsing itself.

\subsection{Meta-Analysis Statistical Approach}

As previously discussed, the conventional meta-analytic method used in the current review is often used within previously published systematic reviews and meta-analyses [49, 51]. However, the conventional approach does not account for the multiple dependent effect sizes (SMDs) from each study. Therefore an adjusted approach using a random effects meta-regression model using robust variance estimation was also used in this current review [67]. As this approach is more conservative, many statistically significant results from the conventional approach were no longer significant at the 5\% level and 95\% confidence intervals crossed zero. It is possible that the moderators included in this review (i.e. sex, oral rinse protocols, exercise protocol, fasting) are potentially associated with the variation of the effect size (SMDs) between studies based on conventional unadjusted results. However, when adjusted using the more conservative approach, results are non-significant. Thus, further independent studies in this area of research may suggest that some of the potential moderators are significantly associated with the variation of the study effect sizes.

\subsection{Strength and Limitations}

This is the first systematic review and meta-analysis to include all performance-based exercise outcomes and also to include only studies that that used a maltodextrin-based, carbohydrate oral rinse. Another strength of the current review is that five databases were systematically searched to ensure all relevant articles were screened and three independent reviewers were used for the screening process to minimise bias. Further strengths are the large pool of studies included for the systematic review $(n=35)$ and meta-analysis $(n=34)$ 
and that due to the complex data in the meta-analysis, the analytical approach accounted for these complexities and limitations.

Several limitations with the studies included in the current review need to be acknowledged. One issue with the data provided within the included studies was the lack of standardisation of exercise protocol or oral rinse protocol among the study methodology. For example, within the pool of studies that examined the effect of carbohydrate oral rising on running performance, the protocol varied widely. The exercise protocols included sprints, time trials and time to exhaustion trials; the carbohydrate oral rinse concentration ranged from 6 to $10 \%$ and the duration of rinsing ranged from 5 to $40 \mathrm{~s}$. This variability makes it difficult to fully understand the mechanisms that may affect exercise performance. It would be beneficial for future research to follow existing, validated exercise protocols to explore the effects of carbohydrate oral rinsing on exercise performance. A further limitation is the complexity of the data set included in the meta-analysis. Synthesising and analysing the data was difficult due to a wide range of exercise protocols, carbohydrate oral rinsing protocols and methodology across the included literature as well as multiple data points in the data set. However, a unique statistical approach was used to account for these issues, in order to minimise caution with interpretation. A further possible limitation to consider is that the $p$-value for the adjusted, conservative, random effects meta-regression model was on the cusp of achieving significance at the 5\% level, with the confidence interval (CI) crossing zero. Consequently, this may indicate the possibility of a higher chance of a type I error.

\subsection{Future Research}

In this field of research, there are areas that remain largely unknown. These areas include the specific effect of maltodextrin structure and origin on maltodextrin-based carbohydrate oral rinsing; the exact mechanism that facilitates improvements in exercise performance after carbohydrate oral rinsing and the precise receptor systems that are involved in this process. Future research investigating carbohydrate oral rinsing and exercise performance should aim to explore these areas further.

Furthermore, one area that has not been considered in previous literature is the participants' individual taste sensitivity to complex carbohydrates. Research has demonstrated that individual taste sensitivity to carbohydrates exists [105, 107-109] and that individual sensitivity to complex carbohydrates can vary largely among individuals [109]. From this research, the question emerges as to whether individual taste sensitivity influences the efficacy of a carbohydrate oral rinse on improving exercise performance. Furthermore, if sensitivity does affect this interaction, what is the degree of effect?

Potentially, individual taste sensitivity may moderate the efficacy of a carbohydrate oral rinse and its effect on improving exercise performance. Research has demonstrated that particular groups of people may experience varying outcomes depending on their levels of carbohydrate taste sensitivity. For example, those who experienced high intensity ratings for complex carbohydrates had larger waist circumferences and a significantly higher daily energy intake in comparison with those who experienced a low intensity [107]. Low et al. [110] also demonstrated that carbohydrate hypersensitive participants consumed $50 \%$ more maltodextrin-based milkshake in comparison with those who were less sensitive and this was independent to their liking of the beverage. These examples illustrate that oral sensitivity and exposure to complex carbohydrates can affect multiple dimensions including consumption and anthropometry. If an individual's sensitivity to complex carbohydrates does influence the magnitude of effect a carbohydrate oral rinse has on exercise performance, this may be a cause of variation in each study.

While research exists on the effects of carbohydrate oral rinsing on exercise performance, no existing literature has explored the effect of carbohydrate taste sensitivity on exercise capacity and performance. Future research should explore this area and examine the effect of individual taste sensitivity on the efficacy of a carbohydrate oral rinse and its subsequent effect on exercise performance.

\subsection{Practical Applications}

Based on the findings of this systematic review and metaanalysis, athletes should consider using a maltodextrinbased, carbohydrate oral rinse to achieve potential improvements in exercise performance.

\section{Conclusions}

Despite conflicting data in the field, this review demonstrates that a maltodextrin-based, carbohydrate oral rinse suggests evidence for improving exercise performance. Although the conventional subgroup analyses demonstrated that a maltodextrin-based, carbohydrate oral rinse was most effective at improving exercise performance when rinsing for $10 \mathrm{~s}$ at a concentration between 6 and $6.5 \%$, this metaanalytic approach was considered to be inaccurate. Alternatively, although non-significant, the more robust, adjusted, meta-regression model suggested some evidence of a maltodextrin-based, carbohydrate oral rinse improving exercise performance overall. 
Supplementary Information The online version contains supplementary material available at https://doi.org/10.1007/s40279-022-01658-3.

\section{Declarations}

Funding Open Access funding enabled and organized by CAUL and its Member Institutions.

Conflict of interest Author C.H., author R.S.J.K., author A.C., author W.L.P.B. and author S.B. declare that they have no potential conflicts of interest that might be relevant to the contents of this manuscript.

Availability of data and material The datasets generated during and/or analysed during the current study are available from the corresponding author on reasonable request.

Authorship statement The authors' responsibilities were as follows: C.H. formulated the research question, conducted the screening of the studies, completed data extraction, completed the quality assessment, completed the risk of bias assessment, completed the meta-analysis and wrote the manuscript. R.S.J.K. was involved with formulating the research question, conducted the screening of the studies, assisted C.H. with writing the manuscript and evaluated the quality assessment and the risk of bias assessment. A.C. was involved with formulating the research question, conducted the screening of the studies and assisted C.H. with writing the manuscript. W.L.P.B. conducted the screening of the studies and assisted C.H. with writing the manuscript. S.J.B. assisted with the completion of the meta-analysis and assisted C.H. with writing the manuscript. All authors read and approved the final manuscript.

Ethics Approval Not applicable.

Consent to Participate Not applicable.

Consent for Publication Not applicable.

Open Access This article is licensed under a Creative Commons Attribution 4.0 International License, which permits use, sharing, adaptation, distribution and reproduction in any medium or format, as long as you give appropriate credit to the original author(s) and the source, provide a link to the Creative Commons licence, and indicate if changes were made. The images or other third party material in this article are included in the article's Creative Commons licence, unless indicated otherwise in a credit line to the material. If material is not included in the article's Creative Commons licence and your intended use is not permitted by statutory regulation or exceeds the permitted use, you will need to obtain permission directly from the copyright holder. To view a copy of this licence, visit http://creativecommons.org/licenses/by/4.0/.

\section{References}

1. Krogh A, Lindhard J. The relative value of fat and carbohydrate as sources of muscular energy: with appendices on the correlation between standard metabolism and the respiratory quotient during rest and work. Biochem J. 1920;14(3-4):290-363. https:// doi.org/10.1042/bj0140290.

2. Bazzucchi I, Patrizio F, Felici F, Nicolò A, Sacchetti M. Carbohydrate mouth rinsing: improved neuromuscular performance during isokinetic fatiguing exercise. Int J Sports Physiol Perform. 2017;12(8):1031-8. https://doi.org/10.1123/ijspp.2016-0583.
3. Coggan AR, Coyle EF. Reversal of fatigue during prolonged exercise by carbohydrate infusion or ingestion. J Appl Physiol (1985). 1987;63(6):2388-95. https://doi.org/10.1152/jappl.1987. 63.6.2388.

4. Coyle EF, Hagberg JM, Hurley BF, Martin WH, Ehsani AA, Holloszy JO. Carbohydrate feeding during prolonged strenuous exercise can delay fatigue. J Appl Physiol Respir Environ Exerc Physiol. 1983;55(1 Pt 1):230-5. https://doi.org/10.1152/jappl. 1983.55.1.230.

5. Burke L, Deakin V. Clinical sports nutrition: sports medicine series. 4th ed. McGraw-Hill Education; 2010.

6. Jeukendrup A. A step towards personalized sports nutrition: carbohydrate intake during exercise. Sports Med. 2014;44(Suppl 1):S25-33. https://doi.org/10.1007/s40279-014-0148-z.

7. Rodriguez NR, Di Marco NM, Langley S, American College of Sports Medicine position stand. Nutrition and athletic performance. Med Sci Sports Exerc. 2009;41(3):709-31. https://doi. org/10.1249/MSS.0b013e31890eb86.

8. Thomas DT, Erdman KA, Burke LM. Position of the Academy of Nutrition and Dietetics, Dietitians of Canada, and the American College of Sports Medicine: Nutrition and Athletic Performance. J Acad Nutr Diet. 2016;116(3):501-28. https://doi.org/10.1016/j. jand.2015.12.006.

9. Burke LM, Hawley JA, Wong SHS, Jeukendrup AE. Carbohydrates for training and competition. J Sports Sci. 2011;29(sup1):S17-27. https://doi.org/10.1080/02640414.2011. 585473.

10. Thomas DT, Burke LM, Erdman KA. Nutrition and athletic performance. Med Sci Sports Exer. 2016;48(3):543-68. https://doi. org/10.1249/mss.0000000000000852.

11. Burke LM, Cox GR, Cummings NK, Desbrow B. Guidelines for daily carbohydrate intake. Sports Med. 2001;31(4):267-99. https://doi.org/10.2165/00007256-200131040-00003.

12. de Oliveira EP, Burini RC. Carbohydrate-dependent, exerciseinduced gastrointestinal distress. Nutrients. 2014;6(10):4191-9. https://doi.org/10.3390/nu6104191.

13. Jeukendrup AE. Carbohydrate feeding during exercise. Eur J Sport Sci. 2008;8(2):77-86. https://doi.org/10.1080/1746139080 1918971.

14. Peters HP, van Schelven FW, Verstappen PA, de Boer RW, Bol E, Erich WB, et al. Gastrointestinal problems as a function of carbohydrate supplements and mode of exercise. Med Sci Sports Exerc. 1993;25(11):1211-24.

15. Peters HP, Wiersma JW, Koerselman J, Akkermans LM, Bol E, Mosterd WL, et al. The effect of a sports drink on gastroesophageal reflux during a run-bike-run test. Int J Sports Med. 2000;21(1):65-70. https://doi.org/10.1055/s-2000-8858.

16. Peters HP, Bos M, Seebregts L, Akkermans LM, van Berge Henegouwen GP, Bol E, et al. Gastrointestinal symptoms in long-distance runners, cyclists, and triathletes: prevalence, medication, and etiology. Am J Gastroenterol. 1999;94(6):1570-81. https://doi.org/10.1111/j.1572-0241.1999.01147.x.

17. Peters HPF, Akkermans LMA, Bol E, Mosterd WL. Gastrointestinal symptoms during exercise. Sports Med. 1995;20(2):65-76. https://doi.org/10.2165/00007256-199520020-00002.

18. Rehrer NJ, Janssen GM, Brouns F, Saris WH. Fluid intake and gastrointestinal problems in runners competing in a $25-\mathrm{km}$ race and a marathon. Int J Sports Med. 1989;10(Suppl 1):S22-5. https://doi.org/10.1055/s-2007-1024950.

19. Chambers ES, Bridge MW, Jones DA. Carbohydrate sensing in the human mouth: effects on exercise performance and brain activity. J Physiol. 2009;587(Pt 8):1779-94. https://doi.org/10. 1113/jphysiol.2008.164285.

20. Devenney S, Collins K, Shortall M. Effects of various concentrations of carbohydrate mouth rinse on cycling performance in a 
fed state. Eur J Sport Sci. 2016;16(8):1073-8. https://doi.org/10. 1080/17461391.2016.1196735.

21. James RM, Ritchie S, Rollo I, James LJ. No dose response effect of carbohydrate mouth rinse on cycling time-trial performance. Int J Sport Nutr Exerc Metab. 2017;27(1):25-31. https://doi.org/ 10.1123/ijsnem.2016-0111.

22. Lambert EV, Gibson AC, Noakes TD. Complex systems model of fatigue: integrative homoeostatic control of peripheral physiological systems during exercise in humans. Br J Sports Med. 2005;39(1):52-62. https://doi.org/10.1136/bjsm.2003.011247.

23. Gibson ASC, Lambert ML, Noakes TD. Neural control of force output during maximal and submaximal exercise. Sports Med. 2001;31(9):637-50. https://doi.org/10.2165/00007256-20013 1090-00001.

24. Kringelbach ML. Food for thought: hedonic experience beyond homeostasis in the human brain. Neuroscience. 2004;126(4):807-19. https://doi.org/10.1016/j.neuroscience. 2004.04.035.

25. Rolls ET. Sensory processing in the brain related to the control of food intake. Proc Nutr Soc. 2007;66(1):96-112. https://doi. org/10.1017/s0029665107005332.

26. Frank GK, Oberndorfer TA, Simmons AN, Paulus MP, Fudge JL, Yang TT, et al. Sucrose activates human taste pathways differently from artificial sweetener. Neuroimage. 2008;39(4):155969. https://doi.org/10.1016/j.neuroimage.2007.10.061.

27. Haase L, Cerf-Ducastel B, Murphy C. Cortical activation in response to pure taste stimuli during the physiological states of hunger and satiety. Neuroimage. 2009;44(3):1008-21. https:// doi.org/10.1016/j.neuroimage.2008.09.044.

28. Carter JM, Jeukendrup AE, Jones DA. The effect of carbohydrate mouth rinse on 1-h cycle time trial performance. Med Sci Sports Exerc. 2004;36(12):2107-11. https://doi.org/10.1249/01. mss.0000147585.65709.6f.

29. Ataide-Silva T, Ghiarone T, Bertuzzi R, Stathis CG, Leandro CG, Lima-Silva AE. CHO mouth rinse ameliorates neuromuscular response with lower endogenous CHO stores. Med Sci Sports Exerc. 2016;48(9):1810-20. https://doi.org/10.1249/mss.00000 00000000973.

30. Bavaresco Gambassi B, de Leal YGSB, Anjos ERPD, Antonelli BA, Goncalves ESDCG, de Montenegro IHPM, et al. Carbohydrate mouth rinse improves cycling performance carried out until the volitional exhaustion. J Sports Med Phys Fit. 2019;59(1):1-5. https://doi.org/10.23736/s0022-4707.17.07980-4.

31. Fares EJ, Kayser B. Carbohydrate mouth rinse effects on exercise capacity in pre- and postprandial States. J Nutr Metab. 2011;2011: 385962. https://doi.org/10.1155/2011/385962.

32. Lane SC, Bird SR, Burke LM, Hawley JA. Effect of a carbohydrate mouth rinse on simulated cycling time-trial performance commenced in a fed or fasted state. Appl Physiol Nutr Metab. 2013;38(2):134-9. https://doi.org/10.1139/apnm-2012-0300.

33. Murray KO, Paris HL, Fly AD, Chapman RF, Mickleborough TD. Carbohydrate mouth rinse improves cycling time-trial performance without altering plasma insulin concentration. J Sports Sci Med. 2018;17(1):145-52.

34. Fraga C, Velasques B, Koch AJ, Machado M, Paulucio D, Ribeiro $\mathrm{P}$, et al. Carbohydrate mouth rinse enhances time to exhaustion during treadmill exercise. Clin Physiol Funct Imaging. 2017;37(1):17-22. https://doi.org/10.1111/cpf.12261.

35. Rollo I, Cole M, Miller R, Williams C. Influence of mouth rinsing a carbohydrate solution on 1-h running performance. Med Sci Sports Exerc. 2010;42(4):798-804. https://doi.org/10.1249/ MSS.0b013e3181bac6e4.

36. Rollo I, Williams C, Gant N, Nute M. The influence of carbohydrate mouth rinse on self-selected speeds during a 30-min treadmill run. Int J Sport Nutr Exerc Metab. 2008;18(6):585-600.
37. Wright BF, Davison G. Carbohydrate mouth rinse improves $1.5 \mathrm{~h}$ run performance: is there a dose-effect? Int J Exerc Sci. 2013;6(4):328-40.

38. Decimoni L, Curty V, Almeida L, Koch A, Willardson J, Machado M. Carbohydrate mouth rinsing improves resistance training session performance. Int J Sports Sci Coach. 2018. https://doi.org/10.1177/1747954118755640.

39. Andersson H, Knight A, Buscombe R, Sinclair J, Edmonds CJ, Bottoms L. The effect of carbohydrate mouth rinse on a 30-minute arm cranking performance. Comp Exerc Physiol. 2016;12(1):41-7. https://doi.org/10.3920/CEP150032.

40. Beelen M, Berghuis J, Bonaparte B, Ballak SB, Jeukendrup AE, van Loon LJ. Carbohydrate mouth rinsing in the fed state: lack of enhancement of time-trial performance. Int J Sport Nutr Exerc Metab. 2009;19(4):400-9. https://doi.org/10.1123/ijsnem.19.4. 400.

41. Cherif A, Meeusen R, Ryu J, Taylor L, Farooq A, Kammoun K, et al. Repeated-sprints exercise in daylight fasting: carbohydrate mouth rinsing does not affect sprint and reaction time performance. Biol Sport. 2018;35(3):237-44. https://doi.org/10.5114/ biolsport.2018.77824.

42. Chong E, Guelfi KJ, Fournier PA. Effect of a carbohydrate mouth rinse on maximal sprint performance in competitive male cyclists. J Sci Med Sport. 2011;14(2):162-7. https://doi.org/10. 1016/j.jsams.2010.08.003.

43. Dorling JL, Earnest CP. Effect of carbohydrate mouth rinsing on multiple sprint performance. J Int Soc Sports Nutr. 2013;10(1):41. https://doi.org/10.1186/1550-2783-10-41.

44. Dunkin JE, Phillips SM. The effect of a carbohydrate mouth rinse on upper-body muscular strength and endurance. J Strength Cond Res. 2017;31(7):1948-53. https://doi.org/10.1519/jsc.00000 00000001668 .

45. Gam S, Guelfi KJ, Fournier PA. Opposition of carbohydrate in a mouth-rinse solution to the detrimental effect of mouth rinsing during cycling time trials. Int J Sport Nutr Exerc Metab. 2013;23(1):48

46. Jeffers R, Shave R, Ross E, Stevenson EJ, Goodall S. The effect of a carbohydrate mouth-rinse on neuromuscular fatigue following cycling exercise. Appl Physiol Nutr Metab. 2015;40(6):557-64.

47. Clark VR, Hopkins WG, Hawley JA, Burke LM. Placebo effect of carbohydrate feedings during a 40-km cycling time trial. Med Sci Sports Exerc. 2000;32(9):1642-7. https://doi.org/10.1097/ 00005768-200009000-00019.

48. de Silva TA, de Di Souza MECA, de Amorim JF, Stathis CG, Leandro CG, Lima-Silva AE. Can carbohydrate mouth rinse improve performance during exercise? A systematic review. Nutrients. 2013;6(1):1-10. https://doi.org/10.3390/nu6010001.

49. Brietzke C, Franco-Alvarenga PE, Coelho-Júnior HJ, Silveira R, Asano RY, Pires FO. Effects of carbohydrate mouth rinse on cycling time trial performance: a systematic review and metaanalysis. Sports Med. 2019;49(1):57-66. https://doi.org/10.1007/ s40279-018-1029-7.

50. de Oliveira JJ, Crisp AH, Barbosa CGR, de Silva AS, Baganha $\mathrm{RJ}$, Verlengia R. Influence of carbohydrate mouth rinse on sprint performance: a systematic review and meta-analysis. J Exer Physiol Online. 2017;20(3):88-99.

51. Pochmuller M, Schwingshackl L, Colombani PC, Hoffmann G. A systematic review and meta-analysis of carbohydrate benefits associated with randomized controlled competition-based performance trials. J Int Soc Sports Nutr. 2016;13:27. https://doi.org/ 10.1186/s12970-016-0139-6.

52. Valenzuela C, Aguilera JM. Effects of maltodextrin on hygroscopicity and crispness of apple leathers. J Food Eng. 2015;144:1-9. https://doi.org/10.1016/j.jfoodeng.2014.07.010.

53. American Chemical Society. Maltodextrin. In: Molecule of the week archive. 2018. https://www.acs.org/content/acs/en/molec 
ule-of-the-week/archive/m/maltodextrin.html. Accessed $07 \mathrm{Apr}$ 2020.

54. BeMiller JN. 19-Carbohydrate and noncarbohydrate sweeteners. In: BeMiller JN, editor. Carbohydrate chemistry for food scientists. 3rd ed. AACC International Press; 2019. p. 371-99.

55. Lim J, Pullicin A. Oral carbohydrate sensing: beyond sweet taste. Physiol Behav. 2019. https://doi.org/10.1016/j.physbeh.2019.01. 021.

56. Liu Q. Understanding starches and their role in foods. In: Cui S, editor. Food Carbohydrates: Chemistry, Physical Properties, and Applications. CRC Press; 2005. p. 309-56.

57. Singh N, Singh J, Kaur L, Sodhi NS, Singh GB. Morphologi$\mathrm{cal}$, thermal and rheological properties of starches from different botanical sources. Food Chem. 2003;81(2):219-31. https://doi. org/10.1016/S0308-8146(02)00416-8.

58. BeMiller JN, Whistler RL. Carboydrates. Third Edition ed. Food Chem. New York: Marcel Dekker; 1996. p. 157-224.

59. Takeiti CY, Kieckbusch TG, Collares-Queiroz FP. Morphological and physicochemical characterization of commercial maltodextrins with different degrees of dextrose-equivalent. Int $\mathbf{J}$ Food Prop. 2010;13(2):411-25. https://doi.org/10.1080/1094291080 2181024.

60. Higgins JPT, Savović J, Page MJ, Elbers RG, Sterne JAC. Cochrane handbook for systematic reviews of interventions cochrane. 2019. www.training.cochrane.org/handbook. Accessed 2 Sept 2020.

61. Shamseer L, Moher D, Clarke M, Ghersi D, Liberati A, Petticrew M, et al. Preferred reporting items for systematic review and meta-analysis protocols (PRISMA-P) 2015: elaboration and explanation. BMJ. 2015;350: g7647. https://doi.org/10.1136/bmj. g7647.

62. Moher D, Liberati A, Tetzlaff J, Altman DG, The PG. Preferred reporting items for systematic reviews and meta-analyses: the PRISMA statement. PLoS Med. 2009;6(7): e1000097. https:// doi.org/10.1371/journal.pmed.1000097.

63. Academy of Nutrition and Dietetics. Quality criteria checklist: primary research. Evidence analysis manual. 2016. https://www. andeal.org/vault/2440/web/files/QCC_3.pdf. Accessed 19 Oct 2020.

64. Boland A, Cherry MG, Dickson R. Doing a systematic review: a student's guide. SAGE; 2014

65. Higgins JPT, Altman DG, Gøtzsche PC, Jüni P, Moher D, Oxman $\mathrm{AD}$, et al. The Cochrane Collaboration's tool for assessing risk of bias in randomised trials. BMJ. 2011;343: d5928. https://doi. org/10.1136/bmj.d5928.

66. Hedges LV, Tipton E, Johnson MC. Robust variance estimation in meta-regression with dependent effect size estimates. Res Synth Methods. 2010;1(1):39-65. https://doi.org/10.1002/jrsm.5.

67. Fisher Z, Tipton E. Robumeta: An R-package for robust variance estimation in meta-analysis. arXiv preprint http://arxiv.org/abs/ 150302220. 2015.

68. Bailey SP, Hibbard J, La Forge D, Mitchell M, Roelands B, Harris GK, et al. Impact of a carbohydrate mouth rinse on quadriceps muscle function and corticomotor excitability. Int J Sports Physiol Perform. 2019;14(7):927-33.

69. Bastos-Silva VJ, Prestes J, Geraldes AAR. Effect of carbohydrate mouth rinse on training load volume in resistance exercises. J Strength Cond Res. 2019;33(6):1653-7. https://doi.org/10.1519/ JSC.0000000000002092.

70. Black CD, Schubert DJ, Szczyglowski MK, Wren JD. Carbohydrate mouth rinsing does not prevent the decline in maximal strength after fatiguing exercise. J Strength Cond Res. 2018;32(9):2466-73.

71. Clarke ND, Kornilios E, Richardson DL. Carbohydrate and caffeine mouth rinses do not affect maximum strength and muscular endurance performance. J Strength Cond Res.
2015;29(10):2926-31. https://doi.org/10.1519/JSC.0000000000 000945.

72. Clarke ND, Hammond S, Kornilios E, Mundy PD. Carbohydrate mouth rinse improves morning high-intensity exercise performance. Eur J Sport Sci. 2017;17(8):955-63.

73. Clarke ND, Thomas JR, Kagka M, Ramsbottom R, Delextrat A. No dose-response effect of carbohydrate mouth rinse concentration on 5-km running performance in recreational athletes. $\mathbf{J}$ Strength Cond Res. 2017;31(3):715-20. https://doi.org/10.1519/ JSC.0000000000001531.

74. Cramer MN, Thompson MW, Périard JD. Thermal and cardiovascular strain mitigate the potential benefit of carbohydrate mouth rinse during self-paced exercise in the heat. Front Physiol. 2015;6:354. https://doi.org/10.3389/fphys.2015.00354.

75. de Oliveira JJ, de Salles AHS, Barbosa CGR, de Silva JAO, de Dias RM, Ribeiro AGSV, et al. Effect of post-activation potentiation and carbohydrate mouth rise on repeated sprint ability in university futsal players. J Exer Physiol Online. 2020;23(2):29-40.

76. Deighton K, Duckworth L, Matu J, Suter M, Fletcher C, Stead S, et al. Mouth rinsing with a sweet solution increases energy expenditure and decreases appetite during $60 \mathrm{~min}$ of self-regulated walking exercise. Appl Physiol Nutr Metab. 2016;41(12):1255-61.

77. Durkin M, Akeroyd H, Holliday A. Carbohydrate mouth rinse improves resistance exercise capacity in the glycogen-lowered state. Appl Physiol Nutr Metab. 2021;46(2):126-32.

78. Green MS, Kimmel CS, Martin TD, Mouser JG, Brune MP. Effect of carbohydrate mouth rinse on resistance exercise performance. J Strength Cond Res. 2020. https://doi.org/10.1519/ JSC.0000000000003755.

79. Jensen M, Stellingwerff T, Klimstra M. Carbohydrate mouth rinse counters fatigue related strength reduction. Int J Sport Nutr Exerc Metab. 2015;25(3):252-61. https://doi.org/10.1123/ ijsnem.2014-0061.

80. Phillips SM, Findlay S, Kavaliauskas M, Grant MC. The influence of serial carbohydrate mouth rinsing on power output during a cycle sprint. J Sports Sci Med. 2014;13(2):252-8.

81. Přibyslavská V, Scudamore EM, Johnson SL, Green JM, Wilcoxson MCS, Lowe JB, et al. Influence of carbohydrate mouth rinsing on running and jumping performance during early morning soccer scrimmaging. Eur J Sport Sci. 2016;16(4):441-7. https:// doi.org/10.1080/17461391.2015.1020345.

82. Rollo I, Homewood G, Williams C, Carter J, Goosey-Tolfrey VL. The influence of carbohydrate mouth rinse on self-selected intermittent running performance. Int J Sport Nutr Exerc Metab. 2015;25(6):550.

83. Rossato LT, Fernandes CTM, Vieira PF, de Branco FMS, Nahas PC, Puga GM, et al. No improvement in running time to exhaustion at $100 \% \mathrm{VO}_{2 \max }$ in recreationally active male runners with a preexercise single-carbohydrate mouth rinse. Int J Sports Physiol Perform. 2019. https://doi.org/10.1123/ijspp.2018-0624.

84. Simpson GW, Pritchett R, O'Neal E, Hoskins G, Pritchett K. Carbohydrate mouth rinse improves relative mean power during multiple sprint performance. Int J Exerc Sci. 2018;11(6):754-63.

85. Sinclair J, Bottoms L, Flynn C, Bradley E, Alexander G, McCullagh S, et al. The effect of different durations of carbohydrate mouth rinse on cycling performance. Eur J Sport Sci. 2014;14(3):259-64. https://doi.org/10.1080/17461391.2013. 785599 .

86. Whitham M, McKinney J. Effect of a carbohydrate mouthwash on running time-trial performance. J Sports Sci. 2007;25(12):138592. https://doi.org/10.1080/02640410601113676.

87. Buist I, Bredeweg SW, Bessem B, van Mechelen W, Lemmink KA, Diercks RL. Incidence and risk factors of running-related injuries during preparation for a 4-mile recreational running 
event. Br J Sports Med. 2010;44(8):598-604. https://doi.org/10. 1136/bjsm.2007.044677.

88. Buist I, Bredeweg SW, van Mechelen W, Lemmink KA, Pepping GJ, Diercks RL. No effect of a graded training program on the number of running-related injuries in novice runners: a randomized controlled trial. Am J Sports Med. 2008;36(1):33-9. https://doi.org/10.1177/0363546507307505.

89. Tonoli C, Cumps E, Aerts I, Verhagen E, Meeusen R. Incidence, risk factors and prevention of running related injuries in longdistance running: a systematic review injury, location and type. Sport en Geneeskd. 2010;5:13-8.

90. Hibbert AW, Billaut F, Varley MC, Polman RCJ. Familiarization protocol influences reproducibility of 20-km cycling time-trial performance in novice participants. Front Physiol. 2017;8:488. https://doi.org/10.3389/fphys.2017.00488.

91. Gant N, Stinear CM, Byblow WD. Carbohydrate in the mouth immediately facilitates motor output. Brain Res. 2010;1350:1518. https://doi.org/10.1016/j.brainres.2010.04.004.

92. Bonsignore MR, Morici G, Abate P, Romano S, Bonsignore G. Ventilation and entrainment of breathing during cycling and running in triathletes. Med Sci Sports Exerc. 1998;30(2):239-45.

93. Garlando F, Kohl J, Koller EA, Pietsch P. Effect of coupling the breathing-and cycling rhythms on oxygen uptake during bicycle ergometry. Eur J Appl Physiol Occup Physiol. 1985;54(5):497501. https://doi.org/10.1007/bf00422959.

94. Abbiss CR, Levin G, McGuigan MR, Laursen PB. Reliability of power output during dynamic cycling. Int J Sports Med. 2008;29(7):574-8. https://doi.org/10.1055/s-2007-989263.

95. Laursen PB, Shing CM, Jenkins DG. Reproducibility of a laboratory-based $40-\mathrm{km}$ cycle time-trial on a stationary wind-trainer in highly trained cyclists. Int J Sports Med. 2003;24(7):481-5. https://doi.org/10.1055/s-2003-42012.

96. Stone MR, Thomas K, Wilkinson M, Gibson ASC, Thompson KG. Consistency of perceptual and metabolic responses to a laboratory-based simulated 4,000-m cycling time trial. Eur J Appl Physiol. 2011;111(8):1807-13. https://doi.org/10.1007/ s00421-010-1818-7.

97. Zavorsky GS, Murias JM, Gow J, Kim DJ, Poulin-Harnois C, Kubow S, et al. Laboratory $20-\mathrm{km}$ cycle time trial reproducibility. Int J Sports Med. 2007;28(9):743-8. https://doi.org/10. 1055/s-2007-964969.

98. Thomas K, Stone MR, Thompson KG, Gibson ASC, Ansley L. Reproducibility of pacing strategy during simulated 20-km cycling time trials in well-trained cyclists. Eur J Appl Physiol. 2012;112(1):223-9. https://doi.org/10.1007/s00421-011-1974-4.

99. Stevens C, Dascombe B. The reliability and validity of protocols for the assessment of endurance sports performance: an updated review. Meas Phys Educ Exerc Sci. 2015;19:177-85. https://doi. org/10.1080/1091367X.2015.1062381.
100. Hurst P, Board E. Reliability of 5-km running performance in a competitive environment. Meas Phys Educ Exer Sci. 2016. https://doi.org/10.1080/1091367X.2016.1233421.

101. Bowtell MV, Tan H, Wilson AM. The consistency of maximum running speed measurements in humans using a feedback-controlled treadmill, and a comparison with maximum attainable speed during overground locomotion. J Biomech. 2009;42(15):2569-74. https://doi.org/10.1016/j.jbiomech.2009. 07.024 .

102. De Smet K, Segers V, Lenoir M, De Clercq D. Spatiotemporal characteristics of spontaneous overground walk-to-run transition. Gait Posture. 2009;29(1):54-8. https://doi.org/10.1016/j.gaitpost. 2008.06.005.

103. Meyer T, Welter JP, Scharhag J, Kindermann W. Maximal oxygen uptake during field running does not exceed that measured during treadmill exercise. Eur J Appl. 2003;88(4-5):387-9. https://doi. org/10.1007/s00421-002-0718-X.

104. Liu C-j, Shiroy DM, Jones LY, Clark DO. Systematic review of functional training on muscle strength, physical functioning, and activities of daily living in older adults. Eur Rev Aging Phys Act. 2014;11(2):95-106. https://doi.org/10.1007/s11556-014-0144-1.

105. Lapis TJ, Penner MH, Lim J. Humans can taste glucose oligomers independent of the hT1R2/hT1R3 sweet taste receptor. Chem Sens. 2016;41(9):755-62. https://doi.org/10.1093/chemse/ bjw088.

106. Arnaoutis G, Kavouras SA, Christaki I, Sidossis LS. Water ingestion improves performance compared with mouth rinse in dehydrated subjects. Med Sci Sports Exerc. 2012;44(1):175-9. https:// doi.org/10.1249/MSS.0b013e3182285776.

107. Low JY, Lacy KE, McBride RL, Keast RS. Carbohydrate taste sensitivity is associated with starch intake and waist circumference in adults. J Nutr. 2017;147(12):2235-42. https://doi.org/10. 3945/jn.117.254078.

108. Lapis TJ, Penner MH, Lim J. Evidence that humans can taste glucose polymers. Chem Sens. 2014;39(9):737-47. https://doi. org/10.1093/chemse/bju031.

109. Low JYQ, Lacy KE, McBride RL, Keast RSJ. Evidence supporting oral sensitivity to complex carbohydrates independent of sweet taste sensitivity in humans. PLoS ONE. 2017;12(12): e0188784. https://doi.org/10.1371/journal.pone.0188784.

110. Low JY, Lacy KE, McBride RL, Keast RS. The associations between sweet taste function, oral complex carbohydrate sensitivity, liking and consumption of ad libitum sweet and non-sweet carbohydrate milkshakes among female adults. Br J Nutr. 2019. https://doi.org/10.1017/s0007114519001703. 


\section{Authors and Affiliations}

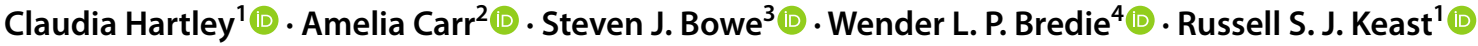

Claudia Hartley

c.hartley@deakin.edu.au

Amelia Carr

amelia.carr@deakin.edu.au

Steven J. Bowe

s.bowe@deakin.edu.au

Wender L. P. Bredie

wb@food.ku.dk

1 CASS Food Research Centre, Deakin University, 221

Burwood Highway, Burwood, VIC 3125, Australia
2 Centre for Sport Research, Deakin University, 221 Burwood Highway, Burwood, VIC 3125, Australia

3 Deakin Biostatistics Unit, Faculty of Health, Deakin University, 221 Burwood Highway, Burwood, VIC 3125, Australia

4 Department of Food Science, University of Copenhagen, Rolighedsvej 26, 1958 Frederiksberg, Denmark 